\title{
Sharp observability estimates for heat equations
}

\begin{abstract}
The goal of this article is to derive new estimates for the cost of observability of heat equations. We develop a new method allowing to show that, when the corresponding wave equation is observable, the heat equation is also observable. This method allows to describe the explicit dependence of the observability constant on the geometry of the problem (the domain in which the heat process evolves and the observation subdomain). We show that our estimate is sharp in some cases, and in particular in one space dimension and in the multi-dimensional radially symmetric case. Our result extends the ones in 12 to the multi-dimensional setting and improves the ones available in the literature, namely those by Miller [28, 30, 31] and Tenenbaum and Tucsnak 39 .
\end{abstract}

This paper has mainly been developed while the first author was a Visiting Fellow of the Basque Center for Applied Mathematics (BCAM). Also partially supported by the Agence Nationale de la Recherche (ANR, France), Project C-QUID number BLAN-3-139579 and Project CISIFS number NT09-437023.

Second author partially supported by the Grant MTM2008-03541 of the MICINN (Spain), project PI2010-04 of the Basque Government, the ERC Advanced Grant FP7-246775 NUMERIWAVES, and the ESF Research Networking Program OPTPDE.

S. Ervedoza

CNRS ; Institut de Mathématiques de Toulouse UMR 5219 ; F-31062 Toulouse, France,

Université de Toulouse ; UPS, INSA, INP, ISAE, UT1, UTM ; IMT ; F-31062

Toulouse, France

E-mail: ervedoza@math.univ-toulouse.fr.

E. Zuazua

IKERBASQUE, Basque Foundation for Science, E-48011 Bilbao - Basque Country - Spain

Basque Center for Applied Mathematics (BCAM), Bizkaia Technology Park, Building 500, E-48160 Derio - Basque Country - Spain

E-mail: zuazua@bcamath.org 
Our approach is based on an explicit representation formula of some solutions of the wave equation in terms of those of the heat equation, contrarily to the standard application of transmutation methods, which uses a reverse representation of the heat solution in terms of the wave one.

We shall also explain how our approach applies and yields some new estimates on the cost of observability in the particular case of the unit square observed from one side. We will also comment the applications of our techniques to controllability properties of heat-type equations.

Keywords Heat equations · Wave equations · Transmutation · Observability cost $\cdot$ Controllability Cost

\section{Introduction}

\subsection{Setting}

The goal of this article is to study the problem of the cost of observability for heat type equations. To fix the ideas, we will mainly consider the classical constant coefficient heat equation although our methods and results apply to a large class of parabolic abstract problems.

Let $\Omega$ be a bounded domain and consider the heat equation with state $z$, solution of

$$
\left\{\begin{array}{lc}
\partial_{t} z-\Delta_{x} z=0, & (t, x) \in \mathbb{R}_{+}^{*} \times \Omega \\
z(t, x)=0, & (t, x) \in \mathbb{R}_{+}^{*} \times \partial \Omega \\
z(0, x)=z_{0}(x), & x \in \Omega
\end{array}\right.
$$

We analyze the problem of observability, which is dual to the controllability one, as we shall explain in Section 3 (see also [23]), and that consists in getting global estimates on the solutions in terms of the energy concentrated on some subdomain of the domain $\Omega$ where the equation evolves.

There is an extensive literature on the subject. In particular, using Carleman inequalities as in [15, one can prove that for any subdomain $\omega \subset \Omega$, there exist constants $C, \gamma>0$ and $\tilde{\gamma}>0$ such that any solution $z$ of the heat equation (1.1) satisfies

$$
\int_{0}^{\infty} \int_{\Omega} \exp \left(-\frac{\gamma}{t}\right)|z(t, x)|^{2} d t d x \leq C \int_{0}^{\infty} \int_{\omega}|z(t, x)|^{2} d t d x,
$$

and for all $T>0$,

$$
\int_{\Omega}|z(T, x)|^{2} d t d x \leq C \exp \left(\frac{\tilde{\gamma}}{T}\right) \int_{0}^{T} \int_{\omega}|z(t, x)|^{2} d t d x .
$$

These are so called observability inequalities that assert that the energy of solutions concentrated in $\omega$ yields an upper bound of the energy everywhere in $\Omega$. For that to happen, because of the strong irreversibility of the heat semigroup, an exponentially vanishing weight is needed at $t=0$ in 1.2 and, similarly, the constant in 1.3 grows exponentially as $T \rightarrow 0$.

The constants $C, \gamma$ and $\tilde{\gamma}$ on the observability inequality 1.2 depend on the geometric properties of $\omega$ and $\Omega$. 
This paper is mainly devoted to the analysis of the constant $\gamma$. Our goal is to prove a new upper bound on the best constant $\gamma$ in $(1.2)$ that, all along this article, will be referred to as being the exponential observability cost. Moreover, this bound will be shown to be sharp in some geometric configurations and in particular in 1-d, a fact that was unknown until now. As we shall explain later in Section 2.4, this constant $\gamma$ characterizes the reachability set for 1.1 .

The constant $\tilde{\gamma}$ is called the finite-time exponential observability cost. Estimates like (1.3) are particularly relevant in small time $T \sim 0$. Note that, according to Lions 23 , estimate $(1.3)$ is equivalent to estimating the cost of null-controllability in time $t=T$, i.e. the norm of the map that, to an initial data $z_{0} \in L^{2}(\Omega)$, associates the control $u$ of minimal $L^{2}((0, T) \times \omega)$-norm such that the solution of

$$
\left\{\begin{array}{lc}
\partial_{t} z-\Delta_{x} z=u(t, x) \chi_{\omega}(x), & (t, x) \in \mathbb{R}_{+}^{*} \times \Omega, \\
z(t, x)=0, & (t, x) \in \mathbb{R}_{+}^{*} \times \partial \Omega, \\
z(0, x)=z_{0}(x), & x \in \Omega .
\end{array}\right.
$$

satisfies $z(T)=0$.

There are several previous results on this subject yielding various lower and upper bounds on $\gamma$ and $\tilde{\gamma}$ that we briefly present below. The first remark is that, obviously,

$$
\gamma \leq \tilde{\gamma}
$$

Lower bounds: The following lower bound on the constant $\tilde{\gamma}$ fulfilling 1.2 was obtained by comparison with the Green function of the heat equation (see 28 ):

$$
\tilde{\gamma} \geq \frac{\tilde{d}^{2}}{2}, \quad \text { with } \tilde{d}=\sup _{x_{0} \in \Omega} d\left(x_{0}, \omega\right) .
$$

Indeed, the Green function centered at a point $x_{0}$ in $\Omega \backslash \omega$ at a geodesic distance $d\left(x_{0}, \omega\right)$ of the observation region $\omega$, which decays as $C t^{-N / 2} \exp (-\mid x-$ $\left.\left.x_{0}\right|^{2} / 4 t\right)$ away from $\omega$, shows that, necessarily, $\tilde{\gamma} \geq d\left(x_{0}, \omega\right)^{2} / 2$ is needed for all $x_{0} \in \Omega$ in order to ensure 1.3 .

On the other hand, in 13,42 , using the functions

$$
z_{\rho}(t, x)=\frac{1}{(4 \pi t)^{n / 2}} \sin \left(\frac{\rho|x|}{2 t}\right) \exp \left(\frac{1}{4 t}\left(\rho^{2}-|x|^{2}\right)\right), \quad \rho>0
$$

solutions of the heat equation in $\mathbb{R}^{N}$, it is shown that

$$
\gamma \geq \frac{d^{2}}{2}, \quad \text { with } d=\sup \left\{\rho, \text { such that } B\left(x_{0}, \rho\right) \subset \Omega \backslash \omega\right\}
$$

Note that, always, $d \leq \tilde{d}$, but in some geometrical situations, $d=\tilde{d}$. This is the case in particular when $\Omega \backslash \omega$ is a ball and $\omega$ is a neighborhood of $\partial \Omega$.

Upper bounds: On the other hand, as mentioned above, Carleman inequalities guarantee that (1.3) holds with a finite constant $\tilde{\gamma}>0$, hence also (1.2) for some constant $\gamma$. But this technique does not provide any explicit 
expression on how the exponential observability constants $\gamma, \tilde{\gamma}$ depend on the geometry of the problem under consideration.

The existing upper bounds refer mainly to the case where the Geometric Control Condition (GCC) is satisfied. GCC asserts that all the rays of Geometric Optics in $\Omega$, reflected according to Descartes' law on the boundary, enter the domain $\omega$ in some finite uniform time $2 S$ (see [4] for a more precise description of the GCC). This imposes, of course, important constraints on the geometry of the control subdomain $\omega$. This condition is sharp in the context of the observability of the wave equation but, as mentioned above, is not needed to establish the observability inequality for the heat equation.

According to [4], the GCC is equivalent to the following observability property for the corresponding wave equation: There exists $C>0$ such that any solution $y$ of the wave system

$$
\begin{cases}\partial_{s s} y-\Delta_{x} y=0, & (s, x) \in \mathbb{R} \times \Omega \\ y=0, & (s, x) \in \mathbb{R} \times \partial \Omega\end{cases}
$$

satisfies

$$
\int_{\Omega}|y(0, x)|^{2} d x+\left\|y_{s}(0, \cdot)\right\|_{H^{-1}(\Omega)}^{2} \leq C \int_{-S}^{S} \int_{\omega}|y(s, x)|^{2} d s d x .
$$

In $(1.9), s$ stands for the time variable of the wave equation, since it is convenient to distinguish it from the time $t$ for the heat process.

The time $2 S$ needed for the GCC to hold, in view of the finite velocity of propagation of waves ( $\equiv 1$ in the present model), is necessarily such that $S \geq \tilde{d}$. This is so since, roughly, in time $2 \tilde{d}$ one can only guarantee that the ray along the geodesic path reaches the observation set, after evolving along a back and forth trajectory, while the GCC requires the same to hold for all the rays. However, there are many cases in which $S>>\tilde{d}$ or even $S$ is infinite. This is precisely the case when $\omega$ fails to satisfy the GCC in any finite time. This happens, for instance, when $\Omega$ is the unit ball and $\omega$ is a ball centered at the origin and of radius $r<1$. However, there are non-trivial situations in which we can guarantee that $S=\tilde{d}=d$, and in particular in the 1-d setting, as we will explain in Section 4.

Under the GCC, it has been shown that the observability inequality (1.3) holds for the heat equation for all

$$
\tilde{\gamma}>\gamma_{*} S^{2} / 2,
$$

with $\gamma_{*}=8(36 / 37)^{2}$ in [28,30]. This upper bound on $\tilde{\gamma}$ was later improved to $\gamma_{*}=3$ in 39 .

As a consequence of this, according to 1.5$)$, the observability inequality (1.2) holds for any $\gamma$ satisfying

$$
\gamma>3 S^{2} / 2
$$

But, even when $S=d$, this upper bound 1.12 on the best observability constant is larger (by a multiplicative factor 3 ) than the lower bound (1.8). 
A sharp result on $\gamma$ in 1-d. The results in 12] imply that, for onedimensional parabolic equations on an interval of length $L$ controlled from one of the points on the boundary, $\gamma$ in $(1.2)$ can be chosen to be any constant strictly larger than $L^{2} / 2$, whereas 1.2 does not hold if $\gamma<L^{2} / 2$.

This is the unique existing result in the literature on the optimality of $\gamma$ in $(1.2)$ as far as we know.

Note that the results in 12 are stated from the point of view of the reachability set of the heat equation but this set is fully determined by the constant $\gamma$ in 1.2 , see Section 2.4

The techniques used in [12] are based on a precise study of the biorthogonal family of $\left(\exp \left(-n^{2} \pi^{2} t\right)\right)_{n \geq 0}$ in $L^{2}(0, T)$, which is not available in higher dimensions.

Also note that this seems to indicate that the lower bound 1.8 for $\gamma$ should be $\tilde{d}^{2} / 2$ instead of $d^{2} / 2$. So far, this is only a conjecture.

As we have said, the fact that the observability property of waves implies the observability of the heat equation is well-known. But this has not been proved directly so far, but rather in the context of the dual equivalent controllability problem. To be more precise, Russell in his pioneer work [37. observed that the exact controllability property of the wave equation implies the null controllability of the corresponding heat process. This, by duality, allows also showing the link between the observability properties of these two models.

The original approach of Russell was based on the method of moments ([37]), and has been more recently modified and replaced by the so-called transmutation method (28,30,31), that has been employed to give the quantitative results on the exponential observability cost mentioned above. Transmutation is easier to apply: it is inspired in Kannai's transform, which allows writing the solutions of the controlled abstract heat equation in terms of the corresponding controlled solutions of the wave model. This approach has been also recently used in 33] to derive an efficient method for numerically computing the control for heat equations.

The main result of this paper ensures that, under the GCC, the observability inequality $(1.2)$ holds for $\gamma=S^{2} / 2$ (or very close variants; see Section 4 for more details). This significantly improves the known estimates (1.12).

According to the lower bound 1.8 , we conclude that our result is sharp when $S=d$. The later is true, as we mentioned above, in one space dimension and in some simple multidimensional geometries: for instance, for any domain $\Omega$ when the control set $\omega$ is a neighborhood of its boundary such that $\Omega \backslash \omega$ is a ball, see Section 4 .

Note that even in the 1 dimensional case, it also improves the results in [12] up to the critical case $\gamma=L^{2} / 2$.

Our approach is also based on a transmutation method, but applied directly on the observability context rather than from the control point of view. The main novelty is that we write solutions of the wave equation as a function of that of the heat equation, in the opposite sense to the classical Kannai transform. This might seem counterintuitive since solutions of the heat equation propagate at an infinite speed, and this could be an obstruction to get the solutions of the wave equation, with a finite velocity of propaga- 
tion. But, in fact, this may be done since our transform maps solutions of the heat equation into a class of analytic solutions of the wave one. Once solutions of the wave equation have been written in terms of those of the heat equation, applying the well known observability properties of the wave equation under the GCC, one recovers observability inequalities for the heat equation with sharp exponential observation cost.

This method will be formulated and presented in an abstract setting containing the heat equation but also other parabolic problems as, for instance, the fourth order diffusion operator.

1.2 The main result

Let $X$ be a Hilbert space and $A$ be a self-adjoint positive definite unbounded operator on $X$ with dense domain $\mathcal{D}(A)$ and compact resolvent.

We then introduce the following abstract heat equation:

$$
\left\{\begin{array}{l}
\partial_{t} z+A z=0, \quad t \in \mathbb{R}_{+} \\
z(0)=z_{0}
\end{array}\right.
$$

and its corresponding wave equation:

$$
\left\{\begin{array}{l}
\partial_{s s} y+A y=0, \quad s \in \mathbb{R} \\
y(0)=y_{0}, \partial_{s} y(0)=y_{1}
\end{array}\right.
$$

The observation is done through an operator $B \in \mathfrak{L}(\mathcal{D}(A), U)$, where $U$ is a Hilbert space.

As we mentioned above, our approach applies under the assumption that the observability property holds for this abstract wave equation as made precise below.

Assumption 1 There exist a time $S>0$ and a constant $C_{w}=C_{\text {wave }}$ such that any solution $y$ of the wave equation (1.14 with initial data $\left(y_{0}, y_{1}\right) \in$ $\mathcal{D}(A) \times \mathcal{D}\left(A^{1 / 2}\right)$ satisfies

$$
\left\|A^{1 / 2} y_{0}\right\|_{X}^{2}+\left\|y_{1}\right\|_{X}^{2} \leq C_{w} \int_{-S}^{S}\|B y(s)\|_{U}^{2} d s
$$

Our main result is the following one:

Theorem 1.1 Let $A$ be a self-adjoint unbounded positive definite operator with dense domain and compact resolvent and $B$ be an observation operator $B \in \mathfrak{L}(\mathcal{D}(A), U)$ such that Assumption 1 holds.

Then there exists $C>0$ such that for any $z$ solution of 1.13 with initial data $z_{0} \in \mathcal{D}(A)$, the following estimate holds

$$
\int_{0}^{\infty} \exp \left(-\frac{S^{2}}{2 t}\right)\|z(t)\|_{X}^{2} d t \leq C \int_{0}^{\infty}\|B z(t)\|_{U}^{2} d t .
$$


Besides, for all $T>0$ there exists $C(T)>0$ such that for any $z$ solution of 1.13 with initial data $z_{0} \in \mathcal{D}(A)$, the following is satisfied:

$$
\int_{0}^{\infty} \exp \left(-\frac{S^{2}}{2 t}\right)\|z(t)\|_{X}^{2} d t \leq C(T) \int_{0}^{T}\|B z(t)\|_{U}^{2} d t .
$$

The following comments are in order:

- In view of this result, one can take any $\gamma>S^{2} / 2$ in 1.2 when the GCC is satisfied, see Section 4 for more details, and even the critical case $\gamma=S^{2} / 2$ provided the norm in the left-hand side of $(1.2)$ is weakened.

- The finite time estimate (1.17) should be made precise further in the sense that it would be interesting to get explicit bounds on how the constant $C(T)$ grows as $T$ tends to zero. This issue is discussed in Section 3.2 and in Section 5

- If $B$ is assumed to be in $\mathfrak{L}\left(\mathcal{D}\left(A^{1 / 2}\right), U\right)$, since $A$ is positive definite, the right hand side of 1.16$)$ is finite for any solution of 1.13 with initial data in $X$. This is so because solutions $z$ of $(1.13)$ with initial data in $X$ belong to $L^{2}\left(\mathbb{R}_{+}, \exp (\nu t) d t ; \mathcal{D}\left(A^{1 / 2}\right)\right)$, for some $\nu>0$ smaller than the first eigenvalue of $A$. Accordingly, when $B \in \mathfrak{L}\left(\mathcal{D}\left(A^{1 / 2}\right), U\right)$, by density, estimate 1.16) can be extended to any $z_{0} \in X$.

If $B$ does not belong to $\mathfrak{L}\left(\mathcal{D}\left(A^{1 / 2}\right), U\right)$ but only to $\mathfrak{L}(\mathcal{D}(A), U)$, one cannot guarantee a priori that the integrals in $(1.16)$ are finite for any initial data $z_{0} \in X$ but the inequalities (1.16)-1.17) make sense for initial data in $\mathcal{D}(A)$.

In Section 3, we will explain how our transmutation technique developed for Theorem 1.1 can be applied directly in a finite-time horizon, using different transmutation kernels that are compactly supported in time $t \in(0, T)$.

In particular, our transmutation method can be used to get a bound on the cost of controllability $\tilde{\gamma}$ in 1.3 (see Section 3.2), though the bound we obtain is worst than the ones in [28,39] when $T \sim 0$. We shall explain why our method fails to improve the bounds in 39.

Our method also identifies an observed quantity for which not only the observability inequality holds but the reverse is also true (see Section 3.3). In other words, we will give an explicit norm on the initial data which is equivalent to some norm of the observation. Note for instance that, although (1.2) holds, the reverse is not true. This issue is of course of particular interest with respect to the control problem, as we explain in Section 3.4. In particular, this can be used to determine a Hilbert Uniqueness Method algorithm to compute smooth controls. This partially explains why transmutation allows to avoid the ill-posedness of the problem of numerically computing the controls (see 33).

We also list a number of examples in which our approach applies. In particular, we focus on the 1-dimensional heat equation. We then consider the case $\Omega=(0,1)^{2}$, the unit square, with observation on the boundary, first when GCC holds, and then when the observation is done only on one side of the unit square. In that later case, though GCC does not hold, transmutation can be applied and also yields in that particular case estimates on the exponential observability cost. 
The outline of the article is as follows. First, in Section 2, we prove Theorem 1.1. We also briefly comment in Section 3 how the techniques we have developed for Theorem 1.1 can be adapted to deal with a finite time horizon, and comment their control theoretical consequences. In Section 4 . we discuss applications of Theorem 1.1 on some examples. Finally, in Section 5. we give some further comments and open problems.

\section{Proof of the main result}

We proceed in several steps that will be presented in different paragraphs.

\subsection{Transmutation : from heat processes to waves}

As we have explained above, transmutation has been so far used to transform results on the control of the wave equation into results on the control of the heat one. For that to be done one has to write the solutions of the heat equation in terms of those of the wave equation in the spirit of the classical Kannai transform (see, for instance, 28]).

But here we apply the transmutation method at the level of the observability property. More precisely, we want to derive observability inequalities for the heat equation as a consequence of the existing observability inequalities for the wave equation. For this to be done one has to write the solutions of the wave equation in terms of those of the heat equation. Such transform is rather counterintuitive since, in view of the finite velocity of propagation underlying the wave operator, it might seem unnatural to try to express its solutions in terms of the heat kernel which diffuses at an infinite speed. But this can be done, indeed, for a suitable class of initial data and this suffices to our purposes.

The key observation of the present article is as follows:

Theorem 2.1 Let $z_{0} \in X$ and $z=z(t)$ be the solution of the abstract heat equation 1.13 with initial datum $z_{0}$.

For any finite $S>0$, the solution of the abstract wave equation 1.14 with initial data

$$
y_{0} \equiv 0, \quad y_{1}=\int_{0}^{\infty} \frac{S}{4 \sqrt{\pi} t^{3 / 2}} \exp \left(-\frac{S^{2}}{4 t}\right) z(t) d t,
$$

in the time interval $-S<s<S$ can be represented as

$$
y(s)=\int_{\mathbb{R}_{+}} \frac{1}{(4 \pi t)^{1 / 2}} \sin \left(\frac{s S}{2 t}\right) \exp \left(\frac{s^{2}-S^{2}}{4 t}\right) z(t) d t .
$$

Proof (Theorem 2.1) Let us consider $z$ the solution of the abstract heat equation (1.13) with initial data $z_{0} \in X$. One can check directly the statement of Theorem 2.1. showing that $y$ given by $(2.2)$ is a solution of (1.14). However, for giving a better insight to the reader, we rather explain how we got this 
result, linking the trajectory $z(t)$ to one of the solutions of the abstract wave equation 1.14.

To do this, we look for a solution $y$ of $(1.14)$ in the form

$$
y(s)=\int_{\mathbb{R}_{+}} k(t, s) z(t) d t,
$$

where $k=k(t, s)$ is a suitable kernel to be made precise below, describing how the wave and heat semigroups are related.

In order to identify the kernel $k$ we formally apply the abstract wave operator to $y$ :

$$
\begin{aligned}
& \partial_{s s} y(s)+A y(s)=\int_{\mathbb{R}_{+}} \partial_{s s} k(t, s) z(t) d t+\int_{\mathbb{R}_{+}} k(t, s) A z(t) d t \\
& =\int_{\mathbb{R}_{+}} \partial_{s s} k(t, s) z(t) d t-\int_{\mathbb{R}_{+}} k(t, s) \partial_{t} z(t) d t \\
& =\int_{\mathbb{R}_{+}} \partial_{s s} k(t, s) z(t) d t+\int_{\mathbb{R}_{+}} \partial_{t} k(t, s) z(t) d t-\lim _{t \rightarrow \infty}(k(t, s) z(t))+k(0, s) z_{0} .
\end{aligned}
$$

This shows that $y$ is a solution of the wave equation 1.14 if $k$ satisfies

$$
\begin{cases}\partial_{t} k+\partial_{s s} k=0, & t \in \mathbb{R}_{+}, s \in \mathbb{R} \\ k(0, s)=0, & s \in \mathbb{R} \\ \lim _{t \rightarrow \infty} k(t, s)=0, & s \in \mathbb{R}\end{cases}
$$

Note that in this system $s$ plays the role of the space variable and that we are dealing with the adjoint heat equation that can be easily transformed into the standard forward one by the change of variables $t \rightarrow-t$.

The existence of such non-trivial kernels $k$ is well known (see, e.g., 18]), even if, of course, problem (2.4) is severely ill-posed. In particular, according to the uniqueness results in [7, if we assume that, for some constant $M$,

$$
|k(t, s)| \leq M \exp \left(M s^{2}\right), \quad t \in \mathbb{R}_{+}, s \in \mathbb{R},
$$

then $k \equiv 0$. Therefore, the solution we are looking for, $k$, has to violate this growth condition.

Note that, formally, for any $k$ satisfying (2.4), we automatically get that $y$ given by (2.3) is a solution of the abstract wave equation (1.14). But for the estimates we will derive later to obtain Theorem 1.1. we will need precise estimates on one such non-trivial kernel $k$.

A key further observation with respect to the constructions in [17, 18, is that, in the present context, we only need the solution $k$ to be defined for $s \in(-S, S)$. We can then look for $k$ satisfying, instead of $(2.4)$, the following restricted system:

$$
\begin{cases}\partial_{t} k(t, s)+\partial_{s s} k(t, s)=0, & t \in \mathbb{R}_{+}, s \in(-S, S) \\ k(0, s)=0, & s \in(-S, S) \\ \lim _{t \rightarrow \infty}|k(t, s)|=0, & s \in(-S, S)\end{cases}
$$


Such $k$ satisfying 2.5 can be given explicitly:

$$
k(t, s)=\frac{1}{(4 \pi t)^{1 / 2}} \sin \left(\frac{s S}{2 t}\right) \exp \left(\frac{s^{2}-S^{2}}{4 t}\right) .
$$

Furthermore, $k$ satisfies the following identities:

$$
k(t, 0)=0, \quad t \in \mathbb{R}_{+}, \quad \partial_{s} k(t, 0)=\frac{S}{4 \sqrt{\pi} t^{3 / 2}} \exp \left(-\frac{S^{2}}{4 t}\right), \quad t \in \mathbb{R}_{+} .
$$

Summarizing, if $z$ is the solution of the abstract heat equation (1.13), then the function $y$ given by 2.3 with this kernel $k$ is precisely a solution of the wave equation 1.14 for $s \in(-S, S)$ with initial data $\left(y_{0}, y_{1}\right)$ as in (2.1). This concludes the proof of Theorem 2.1.

Remark 2.1 Observe also that the function $k$ in $(2.6)$ can be obtained from the Appell transform (see [40]) out of the separated variable solution $v(t, s)=$ $\sin (S s / 2) \exp \left(S^{2} t / 4\right)$ of the adjoint heat equation $\partial_{t} v+\partial_{s s} v=0$.

Also note that this is the same kernel as the one constructed in [13, 42 (see (1.7) ) but with $t$ replaced by $-t$ to switch from the heat operator to the present adjoint one. There, it was used to prove estimates from below for $\gamma$.

\subsection{Observability by transmutation}

Using the transmutation formula of the previous paragraph we can derive a first observability inequality for the heat equation as a consequence of the corresponding one for the wave equation.

The following holds:

Theorem 2.2 Let $B$ be an observation operator $B \in \mathfrak{L}(\mathcal{D}(A), U)$.

If Assumption 1 holds and $A$ is self-adjoint, positive definite and with compact resolvent, for any $z$ solution of 1.13 with initial data $z_{0} \in \mathcal{D}(A)$,

$$
\begin{aligned}
& \left\|\int_{0}^{\infty} \frac{S}{4 \sqrt{\pi} t^{3 / 2}} \exp \left(-\frac{S^{2}}{4 t}\right) z(t) d t\right\|_{X}^{2} \\
& \leq C_{w} \int_{-S}^{S}\left\|\int_{0}^{\infty} \frac{1}{(4 \pi t)^{1 / 2}} \sin \left(\frac{s S}{2 t}\right) \exp \left(\frac{s^{2}-S^{2}}{4 t}\right) B z(t) d t\right\|_{U}^{2} d s,
\end{aligned}
$$

where $C_{w}$ is the constant in 1.15 .

Proof (Theorem 2.2) Let $z_{0} \in \mathcal{D}(A)$ and consider $z(t)$ the corresponding solution to the abstract heat equation 1.13 .

Then Theorem 2.1 yields a solution $y$ of the wave equation on $(-S, S)$, explicitly given through identity 2.2 . Using (1.15), we immediately obtain

$$
\left\|A^{1 / 2} y_{0}\right\|_{X}^{2}+\left\|y_{1}\right\|_{X}^{2} \leq C_{w} \int_{-S}^{S}\left\|\int_{\mathbb{R}_{+}} k(t, s) B z(t) d t\right\|_{U}^{2} d s,
$$

for those initial data $\left(y_{0}, y_{1}\right)$ given by 2.1). This is exactly (2.7). 
Note that, in (2.7), the left hand-side term constitutes a norm on $z_{0}$ whereas the right hand-side one should be estimated in terms of the norm of the observation $B z(t)$. This will be done in the next paragraph.

\subsection{Further estimates}

In view of the estimate (2.7) in Theorem 2.2 in order to get the main result in Theorem 1.1 it is sufficient to estimate the integrals on both sides of (2.7).

For this to be done, it will be convenient to use the spectral decomposition of the functional space $X$ on the basis of the eigenfunctions of $A$ : Since $A$ is a self-adjoint positive definite operator with compact resolvent, its spectrum consists in a sequence of positive eigenvalues $0<\mu_{0} \leq \cdots \leq \mu_{j} \leq \mu_{j+1} \rightarrow \infty$ and an orthonormal (in $X$ ) basis of corresponding eigenvectors $\Phi_{j}$ satisfying $A \Phi_{j}=\mu_{j} \Phi_{j}$.

We now prove classical estimates from below for the left hand side of (2.7) and from above for its right hand side.

Estimates on the left hand side of (2.7).

Lemma 2.1 There exists a constant $C>0$ such that

$$
\int_{0}^{\infty} \exp \left(-\frac{S^{2}}{2 t}\right)\|z(t)\|_{X}^{2} d t \leq C\left\|\int_{0}^{\infty} \frac{1}{t^{3 / 2}} \exp \left(-\frac{S^{2}}{4 t}\right) z(t) d t\right\|_{X}^{2} .
$$

To be more precise, if $z_{0}=\sum a_{j} \Phi_{j}$, solutions $z$ of $\sqrt{1.13}$ with initial data $z_{0}$ satisfy:

$$
\begin{aligned}
& \sum_{j}\left|a_{j}\right|^{2} \frac{\exp \left(-2 S \sqrt{\mu_{j}}\right)}{\left(1+\mu_{j}\right)^{1 / 2}} \leq C\left\|\int_{0}^{\infty} \frac{1}{t^{3 / 2}} \exp \left(-\frac{S^{2}}{4 t}\right) z(t) d t\right\|_{X}^{2}, \\
& \int_{0}^{\infty} \exp \left(-\frac{S^{2}}{2 t}\right)\|z(t)\|_{X}^{2} d t \leq C \sum_{j}\left|a_{j}\right|^{2} \frac{\exp \left(-2 S \sqrt{\mu_{j}}\right)}{\left(1+\mu_{j}\right)^{1 / 2}} .
\end{aligned}
$$

Proof (Lemma 2.1) Expanding $z_{0}$ on the basis $\left(\Phi_{j}\right)$ as $z_{0}=\sum_{j} a_{j} \Phi_{j}$, the corresponding solution $z$ of 1.13 is

$$
z(t)=\sum_{j} a_{j} \Phi_{j} \exp \left(-\mu_{j} t\right) .
$$

This implies in particular that

$$
\left\|\int_{0}^{\infty} \frac{1}{t^{3 / 2}} e^{-\frac{S^{2}}{4 t}} z(t) d t\right\|_{X}^{2}=\sum_{j}\left|a_{j}\right|^{2}\left(\int_{0}^{\infty} \frac{1}{t^{3 / 2}} e^{-\frac{S^{2}}{4 t}-\mu_{j} t} d t\right)^{2} .
$$

We need to determine a lower bound for

$$
F(\mu)=\int_{0}^{\infty} \frac{1}{t^{3 / 2}} \exp \left(-\frac{S^{2}}{4 t}-\mu t\right) d t
$$


For this, set $T_{\mu}=S /(2 \sqrt{\mu})$, and remark that

$$
\begin{aligned}
F(\mu) & \geq \int_{T_{\mu}}^{\infty} \frac{1}{t^{3 / 2}} \exp \left(-\frac{S^{2}}{4 t}-\mu t\right) d t \\
& \geq \exp \left(-\frac{S \sqrt{\mu}}{2}\right) \int_{T_{\mu}}^{\infty} \frac{1}{t^{3 / 2}} \exp (-\mu t) d t \\
& \geq \exp \left(-\frac{S \sqrt{\mu}}{2}\right) \mu^{1 / 2} \int_{\mu T_{\mu}}^{\infty} \frac{1}{t^{3 / 2}} \exp (-t) d t .
\end{aligned}
$$

But, for $\alpha \in[2, \infty)$,

$$
\int_{\alpha}^{\infty} \frac{1}{t^{3 / 2}} \exp (-t) d t=\frac{1}{\alpha^{3 / 2}} \exp (-\alpha)-\frac{3}{2} \int_{\alpha}^{\infty} \frac{1}{t^{5 / 2}} \exp (-t) d t
$$

and

$$
\left|\frac{3}{2} \int_{\alpha}^{\infty} \frac{1}{t^{5 / 2}} \exp (-t) d t\right| \leq \frac{3}{2} \frac{1}{\alpha^{5 / 2}} \int_{\alpha}^{\infty} \exp (-t) d t \leq \frac{3}{4} \frac{1}{\alpha^{3 / 2}} \exp (-\alpha)
$$

which implies in particular, for $\alpha \geq 2$, that

$$
\int_{\alpha}^{\infty} \frac{1}{t^{3 / 2}} \exp (-t) d t \geq \frac{1}{4} \frac{1}{\alpha^{3 / 2}} \exp (-\alpha)
$$

Hence, for $\mu \geq 16 / S^{2}$,

$$
F(\mu) \geq \frac{\exp (-S \sqrt{\mu})}{S \sqrt{2 S} \mu^{1 / 4}} \geq \frac{\exp (-S \sqrt{\mu})}{S \sqrt{2 S}(1+\mu)^{1 / 4}}
$$

For $\mu \in\left[0,16 / S^{2}\right]$, one easily checks that $F$ is continuous and does not vanish. Thus it is bounded from below by some positive constant. We conclude that there exists $c>0$ such that for all $\mu \in \mathbb{R}_{+}$,

$$
F(\mu) \geq c \frac{\exp (-S \sqrt{\mu})}{(1+\mu)^{1 / 4}}
$$

which implies 2.10 by 2.13.

Now, using the same notations as in 2.12 , let us remark that

$$
\int_{0}^{\infty} \exp \left(-\frac{S^{2}}{2 t}\right)\|z(t)\|_{X}^{2} d t=\sum_{j}\left|a_{j}\right|^{2} \int_{0}^{\infty} \exp \left(-\frac{S^{2}}{2 t}-2 \mu_{j} t\right) d t
$$


Estimate 2.11) then follows from the following one: for $\mu \geq \mu_{0}>0$, (recall that $\mu_{0}$ is the smallest eigenvalue of $A$ )

$$
\begin{aligned}
& \int_{0}^{\infty} \exp \left(-\frac{S^{2}}{2 t}-2 \mu t\right) d t=\frac{S}{2 \sqrt{\mu}} \int_{0}^{\infty} \exp \left(-S \sqrt{\mu}\left(t+\frac{1}{t}\right)\right) d t \\
& \leq \frac{S}{2 \sqrt{\mu}} \int_{0}^{3} \exp (-2 S \sqrt{\mu}) d t+\frac{S}{2 \sqrt{\mu}} \int_{3}^{\infty} \exp (-S \sqrt{\mu} t) d t \\
& \leq \frac{S}{2 \sqrt{\mu}}\left(3 \exp (-2 S \sqrt{\mu})+\frac{1}{S \sqrt{\mu}} \exp (-3 S \sqrt{\mu})\right) \\
& \leq C \frac{\exp (-2 S \sqrt{\mu})}{\sqrt{\mu}} \leq C \frac{\exp (-2 S \sqrt{\mu})}{\sqrt{1+\mu}} .
\end{aligned}
$$

Hence, from (2.15)-(2.16), there exists a constant $C$ such that (2.11) holds.

Estimate (2.9) immediately follows from 2.10)-2.11). This concludes the proof of Lemma 2.1 .

Estimates on the right hand side of (2.7).

Lemma 2.2 For all $T>0$, there exists a constant $C_{0}(T)$ such that

$$
\int_{-S}^{S}\left\|\int_{T}^{\infty} k(t, s) B z(t) d t\right\|_{U}^{2} d s \leq C_{0}(T)\left\|\int_{0}^{\infty} \frac{1}{t^{3 / 2}} e^{-\frac{S^{2}}{4 t}} z(t) d t\right\|_{X}^{2} .
$$

Moreover,

$$
\lim _{T \rightarrow \infty} C_{0}(T)=0
$$

Proof (Lemma 2.2) Let $T>0$.

Using that, for some constant $C$ independent of $T>0$,

$$
\begin{aligned}
\int_{T}^{\infty} \frac{d t}{t \log ^{2}(t+2)} & \leq \int_{1}^{\infty} \frac{d t}{t \log ^{2}(t+2)} d t+\int_{\min \{T, 1\}}^{1} \frac{d t}{t \log ^{2}(t+2)} \\
& \leq C\left(\frac{T+1}{T}\right)
\end{aligned}
$$

for each $s \in(-S, S)$, we get

$$
\begin{aligned}
& \left\|\int_{T}^{\infty} k(t, s) B z(t) d t\right\|_{U}^{2} \\
& \leq\left(\int_{T}^{\infty}|k(t, s)|^{2}\|B z(t)\|_{U}^{2} t \log ^{2}(t+2) d t\right) \int_{T}^{\infty} \frac{d t}{t \log ^{2}(t+2)} \\
& \leq C\left(\frac{T+1}{T}\right) \int_{T}^{\infty} \frac{1}{4 \pi t} \sin ^{2}\left(\frac{s S}{2 t}\right) e^{\frac{s^{2}-S^{2}}{2 t}}\|B z(t)\|_{U}^{2} t \log ^{2}(t+2) d t \\
& \leq C\left(\frac{T+1}{T}\right) \int_{T}^{\infty} \log ^{2}(t+2)\|B z(t)\|_{U}^{2} d t .
\end{aligned}
$$


But $B$ belongs to $\mathfrak{L}(\mathcal{D}(A), U)$. Thus,

$$
\int_{T}^{\infty} \log ^{2}(t+2)\|B z(t)\|_{U}^{2} d t \leq C \int_{T}^{\infty}(1+t)^{2}\|z(t)\|_{\mathcal{D}(A)}^{2} d t .
$$

Using the same expansion of the heat solutions as in 2.12 , we obtain

$$
\begin{aligned}
\int_{T}^{\infty}(1+t)^{2}\|z(t)\|_{\mathcal{D}(A)}^{2} d t & \leq \sum_{j}\left|a_{j}\right|^{2} \int_{T}^{\infty}(1+t)^{2} \mu_{j}^{4} \exp \left(-2 t \mu_{j}\right) d t \\
& \leq C \sum_{j}\left|a_{j}\right|^{2} \exp \left(-2 \mu_{j} T\right) \mu_{j}^{3}(1+T)^{2}
\end{aligned}
$$

This shows that, for some $C>0$ independent of $T$,

$$
\int_{-S}^{S}\left\|\int_{T}^{\infty} k(t, s) B z(t) d t\right\|_{U}^{2} d s \leq C \frac{(1+T)^{3}}{T} \sum_{j}\left|a_{j}\right|^{2} \exp \left(-2 \mu_{j} T\right) \mu_{j}^{3} .
$$

Thus, for all $T>0$, setting

$$
\tilde{C}_{0}(T)=\sup _{\mu \geq \mu_{0}}\left\{\exp (-2 \mu T+2 S \sqrt{\mu})(1+\mu)^{1 / 2} \mu^{3} \frac{(1+T)^{3}}{T}\right\},
$$

we have

$$
\sum_{j}\left|a_{j}\right|^{2} \exp \left(-2 \mu_{j} T\right) \mu_{j}^{3} \frac{(1+T)^{3}}{T} \leq \tilde{C}_{0}(T) \sum_{j}\left|a_{j}\right|^{2} \frac{\exp \left(-2 S \sqrt{\mu_{j}}\right)}{\left(1+\mu_{j}\right)^{1 / 2}}
$$

and, obviously, $\lim _{T \rightarrow \infty} \tilde{C}_{0}(T)=0$ because $\mu_{0}$, the smallest eigenvalue of $A$, is strictly positive.

Estimate (2.17) and the limit 2.18 then follow immediately from estimates 2.10, 2.22 and 2.23).

Lemma 2.3 For all $T>0$, there exists a constant $C(T)$ such that

$$
\int_{-S}^{S}\left\|\int_{0}^{T} k(t, s) B z(t) d t\right\|_{U}^{2} d s \leq C \int_{0}^{T}\|B z(t)\|_{U}^{2} d t
$$

Proof (Lemma 2.3) For each $s \in(-S, S)$,

$$
\begin{aligned}
& \left\|\int_{0}^{T} k(t, s) B z(t) d t\right\|_{U}^{2} \\
& \leq \int_{0}^{T}|k(t, s)|^{2}\|B z(t)\|_{U}^{2} \sqrt{t} d t \int_{0}^{T} \frac{d t}{\sqrt{t}} \\
& \leq C \int_{0}^{T} \frac{1}{4 \pi t} \sin ^{2}\left(\frac{s S}{2 t}\right) \exp \left(\frac{s^{2}-S^{2}}{2 t}\right)\|B z(t)\|_{U}^{2} \sqrt{t} d t \\
& \leq C \int_{0}^{T} \frac{1}{\sqrt{t}}\|B z(t)\|_{U}^{2} \exp \left(\frac{s^{2}-S^{2}}{2 t}\right) d t .
\end{aligned}
$$


Besides, for $t \in\left(0, S^{2}\right)$,

$$
\begin{aligned}
& \int_{-S}^{S} \exp \left(\frac{s^{2}-S^{2}}{2 t}\right) d s=2 \int_{0}^{S} \exp \left(\frac{s^{2}-S^{2}}{2 t}\right) d s \\
& =2 \int_{0}^{S-\sqrt{t}} \exp \left(\frac{s^{2}-S^{2}}{2 t}\right) d s+2 \int_{S-\sqrt{t}}^{S} \exp \left(\frac{s^{2}-S^{2}}{2 t}\right) d s \\
& \leq 2 S \exp \left(-\frac{S}{\sqrt{t}}+\frac{1}{2}\right)+2 \sqrt{t} \leq C \sqrt{t},
\end{aligned}
$$

and, obviously, whatever $t>0$ is,

$$
\int_{-S}^{S} \exp \left(\frac{s^{2}-S^{2}}{2 t}\right) d s \leq 2 S .
$$

Combining these two estimates, we deduce that for all $t>0$,

$$
\int_{-S}^{S} \exp \left(\frac{s^{2}-S^{2}}{2 t}\right) d s \leq \min \{2 S, C \sqrt{t}\} .
$$
2.24 .

Thus, integrating 2.25$)$ in $s \in(-S, S)$, we obtain the desired estimate

We are now in position to prove Theorem 1.1

Proof (Theorem 1.1) Combining Theorem 2.2 Lemmas 2.2 and 2.3 we obtain

$$
\begin{aligned}
\left\|\int_{0}^{\infty} \frac{1}{t^{3 / 2}} \exp \left(-\frac{S^{2}}{4 t}\right) z(t) d t\right\|_{X}^{2} \leq C \int_{0}^{T_{0}}\|B z(t)\|_{U}^{2} d t \\
+C C_{0}\left(T_{0}\right)\left\|\int_{0}^{\infty} \frac{1}{t^{3 / 2}} \exp \left(-\frac{S^{2}}{4 t}\right) z(t) d t\right\|_{X}^{2}
\end{aligned}
$$

Taking $T_{0}$ large enough so that

$$
C C_{0}\left(T_{0}\right) \leq 1 / 2
$$

which can be done by Lemma 2.2 , we obtain

$$
\left\|\int_{0}^{\infty} \frac{1}{t^{3 / 2}} \exp \left(-\frac{S^{2}}{4 t}\right) z(t) d t\right\|_{X}^{2} \leq C \int_{0}^{T_{0}}\|B z(t)\|_{U}^{2} d t .
$$

This implies (1.17) for $T_{0}$ from 2.9). Estimates 1.17) for $T \geq T_{0}$ and 1.16) are then straightforward.

To prove (1.17) in any time $T>0$ (and smaller than $T_{0}$ ), we use a compactness argument to show that for all $T>0$, there exists a constant $C$ such that for any $z$ solution of 1.13 with initial data $z_{0}=\sum a_{j} \Phi_{j} \in \mathcal{D}(A)$,

$$
\left\|\int_{0}^{\infty} \frac{1}{t^{3 / 2}} \exp \left(-\frac{S^{2}}{4 t}\right) z(t) d t\right\|_{X}^{2} \leq C \int_{0}^{T}\|B z(t)\|_{U}^{2} d t .
$$


We argue by contradiction.

Fix $T \in\left(0, T_{0}\right)$ and assume that there is no constant $C$ such that 2.29 holds. It would then exist a sequence $z_{n}$ of solutions of 1.13 with initial data $z_{0, n}=\sum_{j} a_{j, n} \Phi_{j} \in \mathcal{D}(A)$ such that

$$
\left\|\int_{0}^{\infty} \frac{1}{t^{3 / 2}} \exp \left(-\frac{S^{2}}{4 t}\right) z_{n}(t) d t\right\|_{X}^{2}=1, \quad \lim _{n \rightarrow \infty} \int_{0}^{T}\left\|B z_{n}(t)\right\|_{U}^{2}=0 .
$$

Note that, using the expansion of $z$ on the basis $\left(\Phi_{j}\right)$, for all $n \in \mathbb{N}$,

$$
\begin{aligned}
\left\|\int_{0}^{\infty} \frac{1}{t^{3 / 2}} \exp \left(-\frac{S^{2}}{4 t}\right) z_{n}(t) d t\right\|_{X}^{2} & =\sum_{j}\left|a_{j, n}\right|^{2} \beta_{j}^{2} \\
\text { where } \beta_{j} & =\int_{0}^{\infty} \frac{1}{t^{3 / 2}} \exp \left(-\frac{S^{2}}{4 t}-\mu_{j} t\right) d t
\end{aligned}
$$

and that, according to estimate 2.14 , for some $C>0$,

$$
\beta_{j} \geq C \frac{\exp \left(-S \sqrt{\mu_{j}}\right)}{\left(1+\mu_{j}\right)^{1 / 4}} .
$$

Thus, $\left(a_{j, n} \beta_{j}\right)$ is bounded in $\ell^{2}(\mathbb{N})$ and, extracting a sequence if necessary, $\left(a_{j, n} \beta_{j}\right)$ weakly converges to some sequence $\left(b_{j} \beta_{j}\right)$ in $\ell^{2}(\mathbb{N})$.

But, due to (2.32), for all $\varepsilon>0$, there exists a constant $c_{\varepsilon}$ such that for all $\left(a_{j}\right) \in \ell^{2}(\mathbb{N})$,

$$
\left\|t \mapsto \sum_{j}\left|a_{j}\right|^{2} \exp \left(-2 \mu_{j} t\right)\right\|_{L^{\infty}(\varepsilon, \infty)} \leq c_{\varepsilon} \sum_{j}\left|a_{j}\right|^{2} \beta_{j}^{2}
$$

This implies in particular that, setting $\tilde{z}(t)=\sum_{j} b_{j} \exp \left(-\mu_{j} t\right) \Phi_{j}$ for all $t>\varepsilon$, $z_{n}$ weakly converges to $\tilde{z}$ in $L^{\infty}(\varepsilon, \infty ; X)$ weak-*. Due to the regularizing effects of the abstract heat equation under consideration, this implies that $z_{n}$ strongly converges to $\tilde{z}$ in $L^{2}(2 \varepsilon, \infty ; \mathcal{D}(A))$ and $z_{n}(2 \varepsilon)$ strongly converges to $\tilde{z}(2 \varepsilon)$ in $\mathcal{D}(A)$.

Therefore, choosing $\varepsilon<T / 3, \tilde{z}(\cdot+2 \varepsilon)$ solves 1.13) with initial data $\tilde{z}(2 \varepsilon)$ and, due to 2.30 and the strong convergence of $z_{n}$ to $\tilde{z}$ in $L^{2}(2 \varepsilon, \infty ; \mathcal{D}(A))$, $B \tilde{z}(t)=0$ for $t \in(2 \varepsilon, T)$. But solutions of $(1.13)$ are analytic in positive time with values in $\mathcal{D}(A)$. Hence $B \tilde{z}(t)=0$ for all $t>2 \varepsilon$ and in particular on $\left(2 \varepsilon, T_{0}+2 \varepsilon\right)$. Applying (1.17) with $T_{0}$ to $\tilde{z}(\cdot+2 \varepsilon)$, we deduce that $\tilde{z}(t) \equiv 0$ for all $t>2 \varepsilon$. Hence the limit sequence $\left(b_{j}\right)$ is identically zero.

But $z_{n}$ strongly converges to $\tilde{z} \equiv 0$ in $L^{2}\left(T, T_{0} ; \mathcal{D}(A)\right)$. Since $B \in$ $\mathfrak{L}(\mathcal{D}(A) ; U)$, we deduce that $B z_{n}$ strongly converges to $B \tilde{z} \equiv 0$ in $L^{2}\left(T, T_{0} ; U\right)$. Consequently, due to 2.30$), B z_{n}$ strongly converges to zero in $L^{2}\left(0, T_{0} ; U\right)$. But then, according to $(2.28),\left(a_{j, n} \beta_{j}\right)$ strongly converges to zero in $\ell^{2}(\mathbb{N})$, which is in contradiction with $(2.30)$.

Hence we have proved $(2.29)$ for any positive time $T>0$.

Estimate (2.9) then yields (1.17) in any time $T>0$ and concludes the proof of Theorem 1.1 . 
Remark 2.2 The regularizing effect of the abstract heat semigroup allows also showing that for all $p>0$ and $\gamma>S^{2} / 2$, any solution $z$ of 1.13 satisfies

$$
\int_{0}^{\infty} \exp \left(-\frac{\gamma}{t}\right)\|z(t)\|_{\mathcal{D}\left(A^{p}\right)}^{2} d t \leq C(\gamma, p) \int_{0}^{\infty}\|B z(t)\|_{U}^{2} d t
$$

with a constant $C=C(\gamma, p)>0$. Indeed, writing $z(t)=\sum_{j} a_{j} \Phi_{j} \exp \left(-\mu_{j} t\right)$ and using (2.16), we get

$$
\int_{0}^{\infty} \exp \left(-\frac{\gamma}{t}\right)\|z(t)\|_{\mathcal{D}\left(A^{p}\right)}^{2} d t \leq C \sum_{j}\left|a_{j}\right|^{2} \mu_{j}^{2 p} \frac{\exp \left(-2 \sqrt{2 \gamma} \sqrt{\mu_{j}}\right)}{\left(1+\mu_{j}\right)^{1 / 2}}
$$

which easily yields the claimed result (2.33) by 2.38 and the estimates (2.32).

Remark 2.3 For convenience, we have assumed that $B$ is bounded from $\mathcal{D}(A)$ to $U$, but our arguments apply similarly when the operator $B$ is unbounded from $\mathcal{D}\left(A^{p}\right)$ to $U$, whatever $p \in \mathbb{N}$ is. The proofs are the same, except for Lemma 2.2 and the compactness argument used in the proof of $(2.29)$, where straightforward modifications need to be applied. This allows to deal with weaker observability properties, such as pointwise observations, as we will explain in Section 4 .

Remark 2.4 It would be interesting to know if the following observability inequality holds: For all $T>0$, there exists a constant $C(T)$ such that solutions $z$ of 1.13 satisfy

$$
\left\|\int_{0}^{\infty} \frac{1}{t^{3 / 2}} \exp \left(-\frac{S^{2}}{4 t}\right) z(t) d t\right\|_{X}^{2} \leq C(T) \int_{-S}^{S}\left\|\int_{0}^{T} k(t, s) B z(t) d t\right\|_{U}^{2} d s
$$

where $k$ is the function given by 2.6 .

Using Theorem 2.2 and Lemma 2.2 , we immediately get that this is true for $T>T_{1}$ for $T_{1}$ large enough.

However, for $T>0$, the compactness argument used in the proof of Theorem 1.1 cannot be applied directly and requires the following unique continuation property: If $z$ denotes a solution of the abstract heat equation (1.13),

$$
\left(\forall s \in(-S, S), \int_{0}^{T} k(t, s) B z(t) d t=0\right) \Longrightarrow \forall t \in(0, T), B z(t)=0 .
$$

Whether or not this unique continuation property holds for any time $T>0$ is an open problem.

Of course, using that $k$ solves $(2.5$, this is equivalent to prove that solutions $y$ of

$$
\partial_{s s} y+A y=-k(T, s) z(T), \quad s \in(-S, S)
$$

with initial data as in (2.1) satisfying $B y(s)=0$ for all $s \in(-S, S)$ vanishes identically. Of course, the source term in 2.37) makes the classical unique continuation results of no use for that particular problem. 
2.4 A first application to control

Let us remark that, under the assumptions of Theorem 1.1. the proof of Theorem 1.1 yields 2.29 . Hence, for any time $T>0$, there exists a constant $C$ such that for all $z$ solution of 1.13 with initial data $z_{0}=\sum a_{j} \Phi_{j}$,

$$
\sum_{j}\left|a_{j}\right|^{2} \beta_{j}^{2} \leq C \int_{0}^{T}\|B z(t)\|_{U}^{2} d t
$$

with $\beta_{j}$ as in 2.31 .

This can be used to show that the reachability space $\mathcal{R}_{T}$, which is the set of all functions that can be obtained as $z(T)$ for $z$ solution of the abstract control system

$$
z^{\prime}+A z=B^{*} u(t), \quad t \geq 0, \quad z(0)=0,
$$

with $u \in L^{2}(0, T ; U)$, contains the set of all data $z_{T}=\sum_{j} a_{j} \Phi_{j}$ satisfying

$$
\sum_{j}\left|a_{j}\right|^{2} \frac{1}{\beta_{j}^{2}}<\infty .
$$

Of course, from the estimates 2.32 , this is implied by

$$
\sum_{j}\left|a_{j}\right|^{2}\left(1+\mu_{j}\right)^{1 / 2} \exp \left(2 S \sqrt{\mu_{j}}\right)<\infty
$$

In a more concise form, this means that $A^{-1 / 4} \exp (-S \sqrt{A}) X \subset \mathcal{R}_{T}$.

Indeed, following 23, 30, let us introduce the functional $J$ defined for $\varphi_{T} \in X$ by

$$
J\left(\varphi_{T}\right)=\frac{1}{2} \int_{0}^{T}\|B \varphi(t)\|_{U}^{2} d t-\left\langle\varphi_{T}, z_{T}\right\rangle_{X},
$$

where $\varphi$ is the solution of the adjoint heat equation

$$
-\partial_{t} \varphi+A \varphi=0, \quad t \in(0, T), \quad \varphi(T)=\varphi_{T} .
$$

Then define the completion $\bar{X}_{T}$ of $\left\{\varphi_{T} \in X\right\}$ with respect to the norm

$$
\left\|\varphi_{T}\right\|_{o b s}^{2}=\int_{0}^{T}\|B \varphi(t)\|_{U}^{2} d t .
$$

Due to estimate 2.38, if $z_{T}=\sum a_{j} \Phi_{j}$ satisfies 2.40, the functional $J$ is well-defined, continuous, convex and coercive in $\bar{X}_{T}$. It therefore has a unique minimizer $\psi_{T} \in \bar{X}_{T}$ which defines a control function $u(t)=B \psi(t)$ (or, more precisely, $u(t)=\mathcal{B} \psi_{T}$, where $\mathcal{B}$ is the unique continuous extension of the map $\varphi_{T} \mapsto B \varphi(t)$ on $\left.\bar{X}_{T}\right)$. As one can check by writing the EulerLagrange equation satisfied by $\psi_{T}$, the corresponding solution $z$ of 2.39 satisfies $z(T)=z_{T}$.

Note that, in [38] (see also 30]), it is proved that the reachability set is independent of $T>0$, which is consistent with the fact that the subspace of the reachability set we have found does not depend on time. 
Remark also that our results improve the ones in [12, where it was proved using biorthogonals that $\exp (-(S+\varepsilon) \sqrt{A}) X \subset \mathcal{R}_{T}$ for any $T>0$ and $\varepsilon>0$ for the case of $1 \mathrm{~d}$ heat equation observed from one boundary.

Indeed, using the estimates in Section 2.3. one can rewrite the results in 12 as follows: one can take any $\gamma>S^{2} / 2$ in $\sqrt{1.2}$ in 1-d when controlling from one boundary. However, the techniques used in 12 are restricted to the 1-d case controlled from one boundary, in which case the control problem can be formulated explicitly as a moment problem.

Therefore other situations (distributed controls in 1-d or any case in higher dimension) do not seem to be handled by the techniques in [12].

Our result also improves some other existing ones in higher dimension, as for instance those in [31, Appendix A], stating that $\exp (-\alpha \sqrt{A}) X \subset \mathcal{R}_{T}$ for any $T>0$ for any $\alpha>4 \sqrt{2}(36 / 37) S$.

\section{Observability and Controllability in finite time}

So far our approach has been presented in an infinite time horizon, in the sense that the transmutation kernel $k$ in 2.6 is not compactly supported in time $t \in \mathbb{R}_{+}$. Below, we explain that there are many possible choices of transmutation kernels, and among them, many that are compactly supported in time $t \in(0, T)$. However, as we shall explain below, they are less explicit as before and therefore the estimates we obtain that way are worst than the ones in the literature. Despite of this, the use of these finite time horizon kernels yields new results for a broad class of abstract heat equations.

\subsection{Transmutation in finite time horizon}

Here, our goal is to show that there are many kernel functions $k(t, s)$, vanishing after some time $T>0$, that can be used to transmute from heat to waves.

Following the proof of Theorem 2.1] given $T>0$, one should then construct a nontrivial solution $k_{T}$ of

$$
\begin{cases}\partial_{t} k_{T}(t, s)+\partial_{s s} k_{T}(t, s)=0, & t \in(0, T), s \in(-S, S), \\ k_{T}(0, s)=0, & s \in(-S, S) \\ k_{T}(T, s)=0, & s \in(-S, S)\end{cases}
$$

Such $k_{T}$ can be constructed following the classical method of Tychonoff (see [17, p.211] and [18]). The idea is to look for a solution $k_{T}$ as a power series expansion in $s$ of the form

$$
k_{T}(t, s)=\sum_{n} \frac{s^{n}}{n !} g_{n}(t)
$$

where the functions $g_{n}$ are smooth and supported on $[0, T]$.

A necessary condition for such expansion to solve 3.1 is to have

$$
g_{2 n}=(-1)^{n} g_{0}^{(n)}, \quad g_{2 n+1}=(-1)^{n} g_{1}^{(n)}, \quad n \in \mathbb{N} .
$$


Such a function $k_{T}$ can be constructed by taking $g_{0}(t) \equiv 0$ and $g_{1}(t)$ of the form

$$
g_{1}(t)= \begin{cases}\exp \left(-\alpha\left(\frac{1}{t}+\frac{1}{T-t}\right)\right), & t \in(0, T), \\ 0 & t \in \mathbb{R} \backslash(0, T),\end{cases}
$$

where $\alpha>0$ is some positive parameter.

It is well-known that $g_{1}$ is a smooth function, but to guarantee the convergence of the power series expansion (3.2), we need more precise estimates, that can be derived using Cauchy's formula (see [17, Pb.3 p.73]):

Lemma 3.1 For each $\delta \in(0,1)$, for all $n \in \mathbb{N}$ and $t \in(0, T)$,

$$
\left|g_{1}^{(n)}(t)\right| \leq \frac{n !}{(\delta \min \{t, T-t\})^{n}} \exp \left(-\frac{\alpha}{(1+\delta) \min \{t, T-t\}}\right)
$$

Proof (Lemma 3.1) Note that, due to the fact that $g_{1}$ is symmetric in $T / 2$, we can restrict ourselves to prove (3.5) only for $t \in(0, T / 2)$.

Fix $t \in(0, T / 2)$. Note that $g_{1}$ is real analytic in a neighborhood of $t$ and can then be extended to an holomorphic function in a neighborhood of $t$, for instance in the ball $B(t, \delta t)$ of center $t$ and radius $\delta t, \delta \in(0,1)$. Thus, the Cauchy formula yields

$$
g_{1}(t)=\frac{1}{2 i \pi} \int_{\Gamma(t, \delta t)} \frac{g_{1}(\tau)}{\tau-t} d \tau
$$

where $\Gamma(t, \delta t)$ denotes the circle of center $t$ and radius $\delta t$.

We then obtain that

$$
g_{1}^{(n)}(t)=\frac{n !}{2 i \pi} \int_{\Gamma(t, \delta t)} \frac{g_{1}(\tau)}{(\tau-t)^{n+1}} d \tau .
$$

Now, explicit computations easily yields that, for $t \in(0, T / 2)$ and $\tau \in \Gamma(t, \delta t)$

$$
\left|g_{1}(\tau)\right|=\exp \left(-\alpha \operatorname{Re}\left(\frac{1}{\tau}+\frac{1}{T-\tau}\right)\right) \leq \exp \left(-\frac{\alpha}{t(1+\delta)}\right),
$$

where $\operatorname{Re}(\tau)$ denotes the real part of $\tau \in \mathbb{C}$ and estimate 3.5 follows immediately.

Lemma 3.1 allows to prove the convergence of the series 3.2 and to obtain the estimate (similarly as in [17, p.212])

$$
\left|k_{T}(t, s)\right| \leq|s| \exp \left(\frac{1}{\min \{t, T-t\}}\left(\frac{s^{2}}{\delta}-\frac{\alpha}{(1+\delta)}\right)\right) .
$$

For (3.8) to be well-defined on $(-S, S)$ for $t \rightarrow 0$ and $t \rightarrow T$ and for $k_{T}$ to solve the time boundary conditions in (3.1), we need that, for some $\delta \in(0,1)$, $\alpha \geq S^{2}(1+1 / \delta)$, that is $\alpha>2 S^{2}$.

We thus have the following: 
Proposition 3.1 For any finite $S>0$, for any $\alpha>2 S^{2}$, there exists a function $k_{T}$ satisfying (3.1) with $k_{T}(t, 0)=0$ and $\partial_{s} k_{T}(t, 0)=g_{1}(t)$ given by (3.4) such that for any $\delta \in(0,1)$ satisfying $\alpha>S^{2}(1+1 / \delta)$, for any $(t, s) \in(0, T) \times(-S, S)$, estimate (3.8) holds and, for all $p \in \mathbb{N}$,

$$
\left|\partial_{t}^{p} k_{T}(t, s)\right| \leq \frac{p !|s|}{(\delta \min \{t, T-t\})^{p}} \exp \left(\frac{1}{\min \{t, T-t\}}\left(\frac{s^{2}}{\delta}-\frac{\alpha}{(1+\delta)}\right)\right) .
$$

Only (3.9) has not been proved, but it follows from Lemma 3.1 and identity 3.2 immediately. Details are left to the reader.

Of course, such $k_{T}$ can be used for transmutation, similarly as in Theorem 2.1

To be more precise, if $z_{0} \in X$ and $z=z(t)$ is the solution of the abstract heat equation 1.13 with initial datum $z_{0}$, the function $y=y(s)$ given by

$$
y(s)=\int_{0}^{T} k_{T}(t, s) z(t) d t, \quad s \in(-S, S)
$$

is a solution of the abstract wave equation 1.14 on $(-S, S)$ with initial data

$$
y_{0} \equiv 0, \quad y_{1}=\int_{0}^{T} \exp \left(-\alpha\left(\frac{1}{t}+\frac{1}{T-t}\right)\right) z(t) d t
$$

Let us finally emphasize that any kernel $k_{T}$ solution of (3.1) can be used for transmutation, which illustrates the flexibility of this approach.

\subsection{Exponential observability cost in finite time}

As we recalled in the introduction, estimates on the cost $\tilde{\gamma}$ of controllability in small time in (1.3) for heat like equations are available in the literature (see 30 39]).

The goal of this paragraph is to explain that our approach also applies to that particular issue, using for instance the function $k_{T}$ given by Proposition 3.1 but, so far, yields a weaker result (but with an easier proof) than the ones in the articles 28, 39. More precisely, we claim that for all solutions of the abstract heat equation (1.13), the finite time observability inequality (1.3) holds with $\tilde{\gamma}>16 S^{2}$ for all $T>0$ with a constant $C$ independent of $T>0$.

This of course follows from the estimate (3.8) and similar estimates as the ones in Section 2.3. The proof is left to the reader.

Let us now explain why this result is so far from the bounds obtained in [30, 39].

This is due to the fact that we have very rough estimates on the function $k_{T}$, which is expected to be highly oscillatory, similarly as $k$ in $(2.6)$. 
In particular, one could look for a solution $k_{\alpha}$ of 2.5 of the form 3.2 with $g_{0}(t)=0$ and

$$
g_{1}(t)=\left\{\begin{array}{l}
\frac{\sqrt{\alpha}}{\sqrt{4 \pi} t^{3 / 2}} \exp \left(-\frac{\alpha}{t}\right), \quad t>0, \quad \alpha>0 . \\
0, \quad t \leq 0,
\end{array}\right.
$$

Though such function $k_{\alpha}$ corresponds to the explicit solution

$$
k_{\alpha}(t, s)=\frac{1}{\sqrt{4 \pi t}} \exp \left(\frac{s^{2} / 4-\alpha}{t}\right) \sin \left(\frac{s \sqrt{\alpha}}{t}\right),
$$

estimates on $g_{1}(t)$ and its derivatives will only yield that, for all $\delta \in(0,1)$,

$$
\left|k_{\alpha}(t, s)\right| \leq|s| \exp \left(\frac{1}{t}\left(\frac{s^{2}}{\delta}-\frac{\alpha}{1+\delta}\right)\right) .
$$

Of course, estimate (3.13) only guarantees the existence of $k_{\alpha}$ for $t \in \mathbb{R}_{+}$and $s \in(-\sqrt{\alpha / 2}, \sqrt{\alpha / 2})$ whereas on the explicit formula $\sqrt{3.12})$, one immediately sees that $k_{\alpha}$ is well-defined on $(t, s) \in \mathbb{R}_{+} \times(-2 \sqrt{\alpha}, 2 \sqrt{\alpha})$.

This indicates that the above estimates do not take into account in a satisfactory way the strong oscillating behavior of the function $k_{\alpha}$ and the conjectured ones of the functions $k_{T}$. This also explains why our technique fails to provide sharp estimates on the finite time exponential observability cost $\tilde{\gamma}$ in 1.3 .

\subsection{Two-sided inequalities}

When dealing with the wave equation, one often obtains two sided inequalities of the following form: There exist some strictly positive constants $c_{w}, C_{w}$, such that any $y$ solution of (1.14) satisfies

$$
c_{w} \int_{-S}^{S}\|B y(s)\|_{U}^{2} d s \leq\left\|A^{1 / 2} y_{0}\right\|_{X}^{2}+\left\|y_{1}\right\|_{X}^{2} \leq C_{w} \int_{-S}^{S}\|B y(s)\|_{U}^{2} d s
$$

This states, in addition to (1.15), an admissibility result, always true when $B \in \mathfrak{L}\left(\mathcal{D}\left(A^{1 / 2}\right), U\right)$, but consequence of a more subtle hidden regularity property when this is not the case (and in particular when considering boundary observation through the normal derivative of solutions for the Dirichlet Laplacian), see e.g. 23.

Inequality (3.14) can be combined with any kernel $k_{T}$ solution of (3.1) (such kernel exists, see Proposition 3.1) to obtain a two-sided observability inequality for the heat equation. To simplify the presentation, we further assume that $k_{T}$ is odd in the variable $s$. (Otherwise, replace $k_{T}$ by $k_{T}(t, s)-$ $k_{T}(t,-s)$.) 
Then the transmutation technique applies and yields:

$$
\begin{aligned}
& c_{w} \int_{-S}^{S}\left\|\int_{0}^{T} k_{T}(t, s) B z(t) d t\right\|_{U}^{2} d s \\
& \leq\left\|\int_{0}^{T} \partial_{s} k_{T}(t, 0) z(t) d t\right\|_{X}^{2} \leq C_{w} \int_{-S}^{S}\left\|\int_{0}^{T} k_{T}(t, s) B z(t) d t\right\|_{U}^{2} d s .
\end{aligned}
$$

Concerning the observed quantity on the initial datum, observe that for $z_{0}=\sum_{j} a_{j} \Phi_{j}$, we have

$$
\begin{aligned}
\left\|\int_{0}^{T} \partial_{s} k_{T}(t, 0) z(t) d t\right\|_{X}^{2}=\sum_{j}\left|a_{j}\right|^{2}\left(\beta_{j}\left(k_{T}\right)\right)^{2}, \\
\text { where } \beta_{j}\left(k_{T}\right)=\int_{0}^{T} \partial_{s} k_{T}(t, 0) \exp \left(-\mu_{j} t\right) d t .
\end{aligned}
$$

We thus define the set of observable states with $k_{T}$ as the Hilbert space given by

$$
\mathcal{O}\left(k_{T}\right)=\left\{z=\sum_{j} a_{j} \Phi_{j}, \quad\|z\|_{\mathcal{O}\left(k_{T}\right)}^{2}=\sum_{j}\left|a_{j}\right|^{2}\left(\beta_{j}\left(k_{T}\right)\right)^{2}<\infty\right\} .
$$

Let us emphasize that this space depends on the kernel transmutation function $k_{T}$.

Rewriting 3.15) using this norm, we deduce that there exist two strictly positive constants $c_{1}, c_{2}$ such that

$$
c_{1}\left\|z_{0}\right\|_{\mathcal{O}\left(k_{T}\right)}^{2} \leq \int_{-S}^{S}\left\|\int_{0}^{T} k_{T}(t, s) B z(t) d t\right\|_{U}^{2} d s \leq c_{2}\left\|z_{0}\right\|_{\mathcal{O}\left(k_{T}\right)}^{2} .
$$

Remark 3.1 The same can be done with the kernel $k$ as in 2.6 , $\beta_{j}$ as in 2.31 and $\mathcal{O}(k)$ as

$$
\mathcal{O}(k)=\left\{z=\sum_{j} a_{j} \Phi_{j}, \quad\|z\|_{\mathcal{O}(k)}^{2}=\sum_{j}\left|a_{j}\right|^{2} \beta_{j}^{2}<\infty\right\},
$$

if $T$ is large enough.

Indeed, according to Remark 2.4 and Lemma 2.2 , for $T_{1}$ large enough, for any $T \geq T_{1}$, for any $z$ solution of the abstract heat equation 1.13 , it holds

$$
c_{1}\left\|z_{0}\right\|_{\mathcal{O}(k)}^{2} \leq \int_{-S}^{S}\left\|\int_{0}^{T} k(t, s) B z(t) d t\right\|_{U}^{2} d s \leq c_{2}\left\|z_{0}\right\|_{\mathcal{O}(k)}^{2} .
$$

Note that in 3.19 , the space $\mathcal{O}(k)$ is independent of the time $T>0$.

But whether or not estimate 3.20 holds in arbitrarily small values of $T>0$ is an open problem, see Remark 2.4. 
Remark 3.2 Let us remark that this is not the first time that one derives such equivalence of norms between an observation and the solutions. Indeed, the by-now classical Fursikov-Imanuvilov's Carleman estimate derived in 15 also yields, for some weights $\eta=\eta(t, x)$ (whose definition is given in an intricate way that reflects the geometrical setting, see [15] for the detailed definition of $\eta$ ), that, given $T>0, \Omega$ and $\omega$, there exists a constant $C$ such all solutions $z$ of 1.1 satisfies

$$
\int_{0}^{T} \int_{\Omega} \eta(t, x)^{2}|z(t, x)|^{2} d t d x \leq C \int_{0}^{T} \int_{\omega} \eta(t, x)^{2}|z(t, x)|^{2} d t d x
$$

and of course, the reverse inequality also holds true.

\subsection{Application to control}

In the sequel, we assume that (3.14) holds for the abstract wave equation, a fact that is well known to be true in many relevant situations. For the solutions of the corresponding heat equation it then follows that the twosided inequalities (3.18) are true. These inequalities can be used to deal precisely with the dual control problem.

A technical assumption. For what follows, it is interesting to further assume that there exists a constant $C$ such that for any $z$ solution of the abstract heat equation (1.13,

$$
\|z(T)\|_{X} \leq C\left\|z_{0}\right\|_{\mathcal{O}\left(k_{T}\right)} .
$$

This is automatically fulfilled in most applications because of the strong regularizing effect of heat-like equations.

Estimate 3.22 means that the map $z_{0} \mapsto z(T)$ is continuous from the set of observable states with $k_{T}$ to $X$. In particular, 3.22 and 3.18 imply

$$
\|z(T)\|_{X}^{2} \leq C \int_{-S}^{S}\left\|\int_{0}^{T} k_{T}(t, s) B z(t) d t\right\|_{U}^{2} d s .
$$

Writing 3.22 on the basis of eigenfunctions of $A$ and recalling the definition (3.17) of the space $\mathcal{O}\left(k_{T}\right)$ and of the coefficients $\beta_{j}\left(k_{T}\right)$, one easily checks that $(3.22$ holds if and only if there exists a constant $C$ such that for all $\mu>0$,

$$
\exp (-\mu T) \leq C \int_{0}^{T} \partial_{s} k_{T}(t, 0) \exp (-\mu t) d t .
$$

Note that the kernel function $k_{T}$ given in Proposition 3.1 satisfies 3.23 (or equivalently 3.22 or 3.25 below): Indeed, when $t \in(0, T) \mapsto \partial_{s} k_{T}(t, 0)$ is non-trivial and non-negative

$$
\int_{0}^{T} \partial_{s} k_{T}(t, 0) e^{-\mu t} d t \geq e^{-\mu T} \int_{0}^{T} \partial_{s} k_{T}(t, 0) d t
$$


and then $C$ in 3.23$)$ can be taken as $C=1 / \int_{0}^{T} \partial_{s} k_{T}(t, 0) d t$.

We emphasize that many of the non-trivial kernels $k_{T}$ solutions of (3.1) satisfy assumption (3.22). This is the case for instance for the kernels $k_{T}$ given by Proposition [3.1. Namely, for any non-trivial non-negative $g_{1}$ such that the expansion 3.2 converges, 3.22 holds using the same arguments as above.

In the following, the transmutation kernel $k_{T}$ solution of (3.1) is fixed and assumed to satisfy (3.22).

The reachability set. Define the reachability set (its name will be justified hereafter)

$$
\mathcal{R}\left(k_{T}\right)=\left\{z=\sum_{j} a_{j} \Phi_{j}, \quad\|z\|_{\mathcal{R}\left(k_{T}\right)}^{2}=\sum_{j}\left|a_{j}\right|^{2} \frac{1}{\left(\beta_{j}\left(k_{T}\right)\right)^{2}}<\infty\right\},
$$

which is the dual space of $\mathcal{O}\left(k_{T}\right)$.

Note that, using this spectral representation of solutions of the heat equation (1.13), one immediately sees that estimate $\sqrt{3.22}$ ) (equivalently $(3.23)$ ) is equivalent to the existence of a constant $C$ such that for any $z$ solution of the abstract heat equation 1.13 ,

$$
\|z(T)\|_{\mathcal{R}\left(k_{T}\right)} \leq C\left\|z_{0}\right\|_{X} .
$$

In particular, this implies that, if $z_{0} \in X$, then $z(T)$ belongs to the reachability set $\mathcal{R}\left(k_{T}\right)$, meaning that all free trajectories of the heat semigroup belong to $\mathcal{R}\left(k_{T}\right)$.

Let us then consider the following control problem: For $z_{0} \in X, z_{T} \in$ $\mathcal{R}\left(k_{T}\right)$, to find a control $u$ so that the solution $z$ of

$$
\partial_{t} z+A z=B^{*} u, \quad t \in(0, T), \quad z(0)=z_{0},
$$

satisfies

$$
z(T)=z_{T}
$$

To deal with this problem, in view of the previous two-sided observability inequalities, following the ideas in Subsection 2.4, we introduce the functional $J$ on $\mathcal{O}\left(k_{T}\right)$ as

$$
\begin{aligned}
J\left(\varphi_{T}\right)=\frac{1}{2} \int_{-S}^{S} \| \int_{0}^{T} k_{T}(T- & t, s) B \varphi(t) d t \|_{U}^{2} d s \\
& +\left\langle\varphi(0), z_{0}\right\rangle_{X}-\left\langle\varphi_{T}, z_{T}\right\rangle_{\mathcal{O}\left(k_{T}\right) \times \mathcal{R}\left(k_{T}\right)},
\end{aligned}
$$

where $\varphi$ is the solution of the adjoint heat equation

$$
-\partial_{t} \varphi+A \varphi=0, \quad t \in(0, T), \quad \varphi(T)=\varphi_{T} .
$$

For convenience, we introduce the free heat equation

$$
\partial_{t} \tilde{z}+A \tilde{z}=0, \quad t \in(0, T), \quad \tilde{z}(0)=z_{0} .
$$


Using this function $\tilde{z}$, multiplying $(3.30)$ by $\varphi$ solution of 3.29 , we immediately get

$$
\left\langle z_{0}, \varphi(0)\right\rangle_{X}=\left\langle\tilde{z}(T), \varphi_{T}\right\rangle_{\mathcal{O}\left(k_{T}\right) \times \mathcal{R}\left(k_{T}\right)} .
$$

Besides, estimate 3.25 implies that

$$
\|\tilde{z}(T)\|_{\mathcal{R}\left(k_{T}\right)} \leq C\left\|z_{0}\right\|_{X} .
$$

Setting

$$
Z_{T}=z_{T}-\tilde{z}(T)
$$

the functional $J$ can be rewritten as

$$
J\left(\varphi_{T}\right)=\frac{1}{2} \int_{-S}^{S}\left\|\int_{0}^{T} k_{T}(T-t, s) B \varphi(t) d t\right\|_{U}^{2} d s-\left\langle\varphi_{T}, Z_{T}\right\rangle_{\mathcal{O}\left(k_{T}\right) \times \mathcal{R}\left(k_{T}\right)} .
$$

Since $Z_{T} \in \mathcal{R}\left(k_{T}\right)$ (see $(3.32)$ ), using $(3.18)$, we deduce that the functional $J$ is continuous and coercive in the space $\mathcal{O}\left(k_{T}\right)$. Since it is strictly convex, it has a unique minimum $\psi_{T} \in \mathcal{O}\left(k_{T}\right)$ which satisfies

$$
\left\|\psi_{T}\right\|_{\mathcal{O}\left(k_{T}\right)} \leq C\left\|Z_{T}\right\|_{\mathcal{R}\left(k_{T}\right)} \leq C\left(\left\|z_{T}\right\|_{\mathcal{R}\left(k_{T}\right)}+\left\|z_{0}\right\|_{X}\right) .
$$

Writing the Euler-Lagrange equation satisfied by $\psi_{T}$, setting, for $s \in$ $(-S, S)$

$$
v(s)=\int_{0}^{T} k_{T}(T-t, s) B \psi(t) d t=B\left(\int_{0}^{T} k_{T}(T-t, s) \psi(t) d t\right),
$$

where $\psi$ is the solution of the abstract heat equation 3.29 corresponding to $\psi_{T}$, we obtain that for all $\varphi_{T} \in \mathcal{O}\left(k_{T}\right)$,

$$
\int_{-S}^{S}\left\langle v(s), \int_{0}^{T} k_{T}(T-t, s) B \varphi(t) d t\right\rangle_{U} d s-\left\langle\varphi_{T}, Z_{T}\right\rangle_{\mathcal{O}\left(k_{T}\right) \times \mathcal{R}\left(k_{T}\right)}=0,
$$

or, equivalently,

$$
\begin{aligned}
\int_{0}^{T}\left\langle\int_{-S}^{S} k_{T}(T-t, s) v(s)\right. & d s, B \varphi(t)\rangle_{U} d t \\
& +\left\langle\varphi(0), z_{0}\right\rangle_{X}-\left\langle\varphi_{T}, z_{T}\right\rangle_{\mathcal{O}\left(k_{T}\right) \times \mathcal{R}\left(k_{T}\right)}=0 .
\end{aligned}
$$

This implies that the function

$$
\left.u(t)=\int_{-S}^{S} k_{T}(T-t, s) v(s) d s, \quad \text { where } v \text { is as in } 3.36\right),
$$

is an admissible control function for $(3.26)$ : Indeed, multiplying 3.26 by $\varphi$ solution of 3.29 , we obtain that, for all $\varphi_{T} \in \mathcal{O}\left(k_{T}\right)$,

$$
\int_{0}^{T}\langle u(t), B \varphi(t)\rangle_{U} d t+\left\langle\varphi(0), z_{0}\right\rangle_{X}-\left\langle\varphi_{T}, z(T)\right\rangle_{\mathcal{O}\left(k_{T}\right) \times \mathcal{R}\left(k_{T}\right)}=0,
$$


which, according to (3.38), implies that $z(T)=z_{T}$.

This control has to have some added advantages with respect to the standard ones since it has been derived using a subtle two-sided observability inequality. In particular, as we describe now, the controls obtained by this method have added regularity properties.

Smoothness of controls. Choosing $\varphi_{T}=\psi_{T}$ in (3.37), we obtain

$$
\int_{-S}^{S}\|v(s)\|_{U}^{2} d s=\left\langle\psi_{T}, Z_{T}\right\rangle_{\mathcal{O}\left(k_{T}\right) \times \mathcal{R}\left(k_{T}\right)} .
$$

Estimates (3.35) and 3.18$)$ then show that

$$
\|v\|_{L^{2}(-S, S ; U)} \leq C\left\|Z_{T}\right\|_{\mathcal{R}\left(k_{T}\right)} \leq C\left(\left\|z_{T}\right\|_{\mathcal{R}\left(k_{T}\right)}+\left\|z_{0}\right\|_{X}\right) .
$$

In view of (3.39) and (3.40), estimates on $k_{T}$ and its time derivatives (in $t$ ) allow to recover estimates on the control $u$ in $H^{k}(0, T ; U)$-norms.

In particular, according to (3.9), for the functions $k_{T}$ constructed in (3.2), for all $p \in \mathbb{N}, \partial_{t}^{p} k_{T} \in L^{\infty}((0, T) \times(-S, S))$. Therefore, the control function $u$ in (3.39) satisfies the following: For all $p \in \mathbb{N}$, there exist constants $C_{p, 1}, C_{p, 2}$ such that

$$
\begin{aligned}
\|u\|_{H^{p}(0, T ; U)} \leq & C_{p, 1}\|v\|_{L^{2}(-S, S ; U)} \\
& \leq C_{p, 2}\left\|Z_{T}\right\|_{\mathcal{R}\left(k_{T}\right)} \leq C_{p, 2}\left(\left\|z_{T}\right\|_{\mathcal{R}\left(k_{T}\right)}+\left\|z_{0}\right\|_{X}\right) .
\end{aligned}
$$

Note that this result is specific to the controls we have constructed using the kernels $k_{T}$. Indeed, the recent results in [26] show that the classical controls of minimal $L^{2}(0, T ; U)$-norm fail to have such a property.

However, remark that the controls constructed in 15 using a minimization process of a functional based on the Carleman weights also enjoy nice regularity properties. We refer to 15 for precise statements in that direction for heat equations, and to [8, Propositions 2 and 3] for the Stokes equations.

To better understand the nature of the control for the heat equation constructed by minimization of the functional $J$ in (3.28), we analyze in more detail the function $v$ in $(3.36$.

For $\varphi_{T}=\sum_{j} a_{j} \Phi_{j}$, setting $y(s)=\int_{-S}^{S} k_{T}(T-t, s) \varphi(t) d t, y(0)=0$ and $\partial_{s} y(0)=\sum_{j} a_{j} \Phi_{j} \beta_{j}\left(k_{T}\right)$, identity (3.37) reads as

$$
\int_{-S}^{S}\langle v(s), B y(s)\rangle_{U} d s-\left\langle\partial_{s} y(0), Y_{0}\right\rangle_{X}=0,
$$

where $Y_{0}$ is given by

$$
Y_{0}=\sum_{j} \frac{z_{j}}{\beta_{j}\left(k_{T}\right)} \Phi_{j}, \quad \text { for } Z_{T}=\sum_{j} z_{j} \Phi_{j}
$$

Remark that

$$
\left\|Y_{0}\right\|_{X}^{2}=\left\|Z_{T}\right\|_{\mathcal{R}\left(k_{T}\right)}^{2}, \quad\left\|\partial_{s} y(0)\right\|_{X}^{2}=\left\|\varphi_{T}\right\|_{\mathcal{O}\left(k_{T}\right)}^{2} .
$$


Therefore, the map

$$
\varphi_{T}=\sum_{j} a_{j} \Phi_{j} \mapsto \partial_{s} y(0)=\sum_{j} a_{j} \Phi_{j} \beta_{j}\left(k_{T}\right)
$$

is an isomorphism from $\mathcal{O}\left(k_{T}\right)$ to $X$, and 3.42 is satisfied for any $y$ solution of (1.14) with initial data $\left(y(0), \partial_{s} y(0)\right)=\left(y_{0}, y_{1}\right) \in\{0\} \times X$.

by

Besides, according to $(3.36), v$ can be written as $B \tilde{y}$, where $\tilde{y}(s)$ is given

$$
\tilde{y}(s)=\int_{0}^{T} k_{T}(T-t, s) \psi(t) d t .
$$

Of course, due to the properties of the kernel $k_{T}, \tilde{y}$ is a solution of the abstract wave equation (1.14) with initial data $\left(0, \partial_{s} \tilde{y}(0)\right) \in\{0\} \times X$.

by

Hence, by (3.42), $\tilde{y}_{1}=\partial_{s} \tilde{y}(0)$ is a critical point of the functional $\tilde{J}$ defined

$$
\tilde{J}\left(y_{1}\right)=\frac{1}{2} \int_{-S}^{S}\|B y(s)\|_{U}^{2} d s-\left\langle y_{1}, Y_{0}\right\rangle_{X},
$$

for $y_{1} \in X$, where $y$ is the solution of

$$
\partial_{s s} y+A y=0, \quad s \in(-S, S), \quad\left(y(0), \partial_{s} y(0)\right)=\left(0, y_{1}\right) .
$$

Due to (3.14), the functional $\tilde{J}$ is continuous, coercive and strictly convex in $X$, and then has a unique minimizer, given by $\tilde{y}_{1}$.

To sum up, $v$, extended as an odd function on $(-S, S)$, can be computed on $(0, S)$ by minimization of a suitable functional $\tilde{J}$ defined entirely on the wave equation.

Actually, the function $v(s)$ can also be viewed as the control of minimal $L^{2}(0, S ; U)$-norm such that the solution $Y$ of

$$
\left\{\begin{array}{l}
\partial_{s s} Y+A Y=2 B^{*} v, \quad s \in \mathbb{R} \\
Y(S)=0, \partial_{s} Y(S)=0
\end{array}\right.
$$

satisfies the control requirement

$$
Y(0)=Y_{0}, \quad \text { where } Y_{0} \text { is given by } 3.43 \text {. }
$$

To see that, first remark that, when $y_{0}=0$, solutions $y$ of (1.14) are odd in the time variable $s$. Thus, $\tilde{J}$ can be written as

$$
\tilde{J}\left(y_{1}\right)=\int_{0}^{S}\|B y(s)\|_{U}^{2}-\left\langle y_{1}, Y_{0}\right\rangle_{X} .
$$

Writing the Euler-Lagrange equation satisfied by $\tilde{J}$ at $\tilde{y}_{1}$, one easily derives that $Y$ solution of (3.46) with $v=B \tilde{y}$ satisfies (3.47).

Once the control $v$ of the abstract wave equation is characterized in this manner, the results obtained in [10] can be easily modified to deal with this case (using in particular that, for any $\tau>0$, if $y$ is a solution of 1.14 with $y_{0}=0$, so is $y_{\tau}(s)=(y(s+\tau)-2 y(s)+y(s-\tau)) / \tau^{2}$ since $y$ is odd $)$. In particular, when $B$ belongs to $\mathfrak{L}\left(\mathcal{D}\left(A^{1 / 2}\right), U\right)$ and $B^{*} B \in \cap_{p>0} \mathfrak{L}\left(\mathcal{D}\left(A^{p}\right)\right)$ 
(otherwise, a time-dependent smooth weight function $\eta(s)$ should be introduced within the functional $J$ in (3.28), see [10]), it follows that for all $\ell \geq 0$, there exists a constant $C_{\ell}$ such that

$$
\|v\|_{H^{\ell}(-S, S ; U)}+\left\|A^{\ell} \tilde{y}_{1}\right\|_{X} \leq C_{\ell}\left\|A^{\ell} Y_{0}\right\|_{X}=C_{\ell}\left\|A^{\ell} Z_{T}\right\|_{\mathcal{R}\left(k_{T}\right)} .
$$

We emphasize that 3.48 concerns the regularity properties of $v=v(s)$. The control $u$ for the heat equation given by $(3.39)$ is always smooth in time provided the functions $\partial_{t}^{p} k_{T}$ all belong to $L^{\infty}((0, T) \times(-S, S))$, without these extra regularity assumptions on $B$ (see (3.41)).

Also note that, as explained in [10, the extra time-regularity properties of $v$ imply extra space regularity properties.

To sum up, we have proved the following:

Theorem 3.1 Let $T>0$ and $k_{T} \in L^{\infty}((0, T) \times(-S, S))$ be a solution of (3.1) satisfying (3.22). Assume that (3.14) holds for solutions of the abstract wave equation (1.14). Let $z_{0} \in X$ and $z_{T} \in \mathcal{R}\left(k_{T}\right)$ (defined in (3.24)).

Construction of the control. The functional $J$ in 3.28 has a unique minimizer $\psi_{T}$ on $\mathcal{O}\left(k_{T}\right)$ (defined in $(3.17)$ ), which yields a control u solving the control problem 3.26)-3.27, given by

$$
u(t)=\int_{-S}^{S} \int_{0}^{T} k_{T}(T-t, s) k_{T}(T-\tau, s) B \psi(\tau) d \tau d s,
$$

and there exists a constant $C$ such that

$$
\left\|\psi_{T}\right\|_{\mathcal{O}\left(k_{T}\right)}+\|u\|_{L^{2}(0, T ; U)} \leq C\left(\left\|z_{0}\right\|_{X}+\left\|z_{T}\right\|_{\mathcal{R}\left(k_{T}\right)}\right) .
$$

Another way to compute the control $u$ is the following: Find the minimizer $\tilde{y}_{1} \in X$ of the functional $\tilde{J}$ defined on the waves (with $Y_{0}$ as in $(3.43)-(3.33)$ ), set $v(s)=B \tilde{y}(s)$. Then the control function $u$ is given by (3.39).

Smoothness properties of the control function.

- For any $p \in \mathbb{N}$, if $\partial_{t}^{p} k_{T} \in L^{\infty}((0, T) \times(-S, S))$, u belongs to $H^{p}(0, T ; U)$ and satisfies 3.41.

- If for some $\ell \in \mathbb{N}, A^{\ell} Z_{T} \in \mathcal{R}\left(k_{T}\right)$ and $B B^{*} \in \cap_{p \leq \ell} \mathfrak{L}\left(\mathcal{D}\left(A^{p}\right)\right)$, then $A^{\ell} \tilde{y}_{1} \in X$, 3.48 holds and $v$ belongs to the space

$$
V_{\ell}=\bigcap_{p=0}^{\ell} C^{p}\left([-S, S] ; B^{*} \mathcal{D}\left(A^{\ell-p}\right)\right) .
$$

This automatically yields the following corollary:

Corollary 3.1 Under the assumptions of Theorem 3.1 and with the same notations, if for all $p \in \mathbb{N}, \partial_{t}^{p} k_{T} \in L^{\infty}((0, T) \times(-S, S)), B B^{*} \in \cap_{p \in \mathbb{N}} \mathfrak{L}\left(\mathcal{D}\left(A^{p}\right)\right)$, and for some $\ell \in \mathbb{N}, A^{\ell} Z_{T} \in \mathcal{R}\left(k_{T}\right)$, the source term $B^{*} u$ satisfies

$$
B^{*} u \in C^{\infty}\left([0, T] ; \mathcal{D}\left(A^{\ell}\right)\right)
$$

and therefore $Z=z-\tilde{z}$, with $z$ solution of the control problem $(3.26)-(3.27)$ and $\tilde{z}$ as in 3.30 , solution of the control problem

$$
Z^{\prime}+A Z=B^{*} u, \quad t \in(0, T), \quad Z(0)=0, \quad Z(T)=Z_{T},
$$

satisfies

$$
Z \in C^{\infty}\left([0, T] ; \mathcal{D}\left(A^{\ell+1 / 2}\right)\right)
$$


All the results in this Corollary except for the regularity property (3.54) have been already explained. This latest statement can be deduced immediately from the regularity 3.52 of the source term in 3.53 by induction (see also [10, Corollary 1.5] where similar results are obtained). Details of the proof of Corollary 3.1 are left to the reader.

Note that the regularity result in $(3.54)$ concerns $Z$, the solution of 3.53 . To recover the controlled trajectory $z$ solution of $(3.26)-(3.27)$, one has to add $Z$ and $\tilde{z}$, solution of 3.30 , whose regularity depends only on the initial data to be controlled $z_{0} \in X$. In particular, if $z_{0}$ only is in $X, \tilde{z}$ cannot be continuous on $[0, T]$ with values in $\mathcal{D}(A)$, despite the parabolic regularization effects. That explains why we need to decouple the regularity properties coming from the initial data from the ones coming from the control.

The regularity results in Theorem 3.1 indicate that this control $u$, obtained through two-sided observability inequalities, and characterized as the minimizer of the quadratic functional (3.28), could be of use to avoid the numerical ill-posedness of the effective computation of the controls of the heat equation (see 33]). This subject needs of further investigation.

Actually the method of transmutation of [30] has been already used in 33 . to derive effective methods for computing the controls of the heat equation. But there it has been applied in the classical manner, following [30], writing the controls of the heat equation in terms of those of the wave one, but not as in the present paper, exploiting the new two-sided observability inequalities we have derived here.

Let us also emphasize that the controls given by our approach and the ones provided by the method in [30] are different. Indeed, the transmutation technique used in 30] consists in writing the trajectories of the heat in terms of the waves. We are doing the reverse. Hence the conditions on the transmutation kernels in [30] are different than ours. For instance, in our case, the control function $v$ needs only to control one component of the wave equation. Also note that the control given by the transmutation of [30] is based on a null-control for the wave equation with initial data $z_{0}$, whereas with our approach, it is based on a control for the wave equation with the data $Y_{0}$ (in the sense of (3.46)-(3.47) ) given by (3.43), hence constructed using $Z_{T}$ and the kernel $k_{T}$. Roughly speaking, this explains why our method has more flexibility than the one presented in 30 .

Remark 3.3 Theorem 3.1 and Corollary 3.1 still hold with $k$ as in $(2.6)$ when the time $T$ is large enough, since the key estimate 3.20 holds for $T$ large enough (see Remark 3.1), and $k$ obviously satisfies (3.22).

However, whether or not Theorem 3.1 with $k$ as in 2.6 holds in any finite time $T>0$ is an open problem, see Remark 2.4 .

\section{Examples}

4.1 The 1-dimensional case

Internal observation. Let $\Omega=(0,1)$ and $\omega=\left(\alpha_{1}, \alpha_{2}\right)$ be a non-empty subinterval. Define $X=H^{-1}(\Omega)=H^{-1}(0,1), A=-\Delta$ with domain 
$\mathcal{D}(A)=H_{0}^{1}(\Omega)=H_{0}^{1}(0,1)$, and $B=\chi_{\omega}$, where $\chi_{\omega}$ is the characteristic function of the set $\omega$. Then $B$ is continuous from $\mathcal{D}\left(A^{1 / 2}\right)=L^{2}(\Omega)=L^{2}(0,1)$ to $U=L^{2}(\omega)$.

In this case, it is classical that the wave equation is observable in any time $2 S$ with $S>\tilde{d}=\max \left\{\alpha_{1}, 1-\alpha_{2}\right\}$, see for instance [23].

Applying Theorem 1.1. for any $S>\tilde{d}=\max \left\{\alpha_{1}, 1-\alpha_{2}\right\}$, we automatically get that any solution $z$ of the heat equation (1.1) with initial data $z_{0} \in H^{-1}(0,1)$ satisfies

$$
\int_{0}^{\infty} \exp \left(-\frac{S^{2}}{2 t}\right)\|z(t)\|_{H^{-1}(0,1)}^{2} d t \leq C \int_{0}^{\infty} \int_{\omega}|z(t)|^{2} d t d x,
$$

for some $C>0$ independent of the initial data $z_{0}$.

Of course, this is not exactly (1.2) since the norm in the left hand-side is the $H^{-1}(0,1)$-norm instead of the $L^{2}(0,1)$-one.

Using Remark 2.2 with $p=1 / 2$, we immediately get 1.2 for any $\gamma>$ $\tilde{d}^{2} / 2=\max \left\{\alpha_{1}, 1-\alpha_{2}\right\}^{2} / 2$.

There is no evidence so far that this result is sharp since the lower bound (1.8) yields only $\gamma \geq d^{2} / 2=\tilde{d}^{2} / 8$.

However, when $\omega=\left(0, \alpha_{1}\right) \cup\left(\alpha_{2}, 1\right)$, our results applies and yields 4.1$)$ for any $S>d=\tilde{d}=\left(\alpha_{2}-\alpha_{1}\right) / 2$, which is sharp from the lower bound 1.8$)$ on the observability constant in 1.2 .

Boundary observation. Again, let $\Omega=(0,1)$. Define $X=L^{2}(\Omega), A=-\Delta$ with domain $\mathcal{D}(A)=H^{2} \cap H_{0}^{1}(\Omega)=H^{2} \cap H_{0}^{1}(0,1)$, and $B$ from $\mathcal{D}(A)$ to $U=\mathbb{R}$ given by $B z=\partial_{x} z(x=1)$.

In this context the classical results on the observability of the wave equation (see, e.g., 23]) show that the corresponding wave equation is observable in time $2 S=2$.

Applying Theorem 1.1. we immediately get that any solution $z$ of the heat equation (1.1) with initial data $z_{0} \in H^{1}(0,1)$ satisfies

$$
\int_{0}^{\infty} \int_{0}^{1} \exp \left(-\frac{1}{2 t}\right)|z(t, x)|^{2} d t d x \leq C \int_{0}^{\infty}\left|\partial_{x} z(t, 1)\right|^{2} d t .
$$

Due to [12, estimate 4.2 is sharp.

\subsection{The multi-dimensional case}

Optimality of the results. The 1-dimensional examples above can be easily extended to the multi-dimensional setting. Indeed, given any domain $\Omega$, if $\omega$ is a neighborhood of its boundary such that $\Omega \backslash \omega$ is a ball of radius $R$, the GCC (in time $2 S$ ) holds for any $S>R$ (again, see, for instance, 23]), whereas $d=\tilde{d}=R$.

Applying Theorem 1.1 the lower bound 1.8 on the observability constant in 1.2 is shown to be sharp in this case. We conclude that the observability constant $\gamma$ in 1.2 can be taken to be any constant larger than $d^{2} / 2=\tilde{d}^{2} / 2$. 
Non optimal results. Note that it is easy to build multi-dimensional examples in which GCC holds but $S>\tilde{d}>d$, or in which simply GCC does not hold.

The unit square observed from two consecutive sides. Let $\Omega=$ $(0,1)^{2}$ be the unit square and observe the normal derivative of the solution on two consecutive sides of its boundary, see Figure 1, left. In that case, GCC holds, and $S$ can be taken to be any constant larger than $\sqrt{2}$ but $\tilde{d}=1$ and $d=1 / 2$. Thus, the bounds we get on the observability constant in $(1.2)$ are of the form

$$
\frac{1}{8}=\frac{d^{2}}{2} \leq \gamma \leq \frac{S^{2}}{2}=1^{+}, \quad \text { whereas } \frac{\tilde{d}^{2}}{2}=\frac{1}{2} .
$$

Although this improves the previously existing results, it does not produce a complete identification of the best observability constant.

This example shows that, even if the GCC holds, the direct application of the approach of this paper, using the transformation from waves to heat equations, cannot yield in general a sharp upper bound.
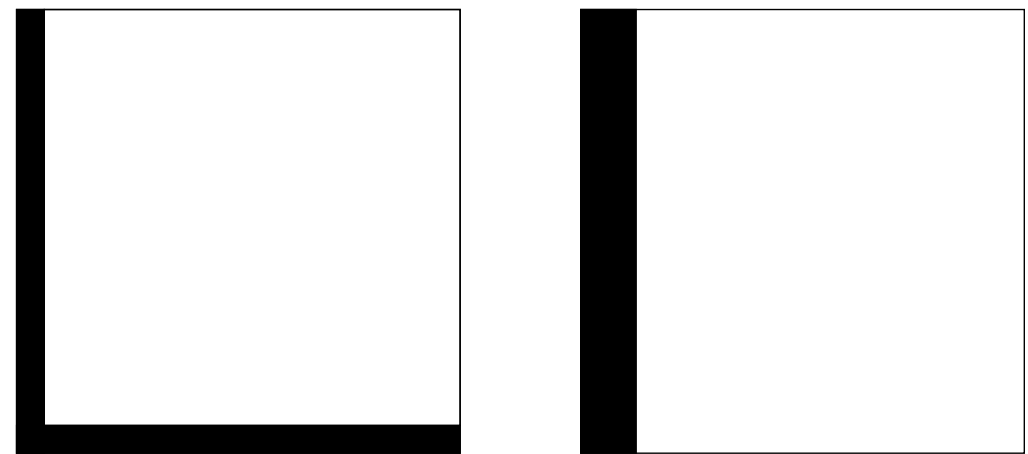

Fig. 1 Squares with and without GCC: In bold, the observation region. Left, GCC holds in time $2 S=2 \sqrt{2}$. Right, GCC does not hold.

The unit square observed from only one side. Here, we consider the heat equation in the unit square $\Omega=(0,1)^{2}$, observed from one side of the boundary $\Gamma=\{0\} \times(0,1)$, see Figure 1 right.

In that case, the wave equation is not observable, since any vertical line corresponds to a trapped ray that does not meet the control region $\Gamma$.

Though, as we will see next, in this very precise situation, our approach can be slightly modified to yield some estimates on the exponential observability cost.

\subsection{Some further examples}

Theoretical remarks. The transmutation technique developed in Section 2 also applies in the context of very weak observability properties. 
To be more precise, one could replace Assumption 1 by the following one: There exists a norm $\|\cdot\|_{*}$ and a time $\tilde{S}>0$ such that the following weak observability inequality is satisfied: there exists a constant $C$ such that for any solution $y$ of 1.14 with initial data $\left(y_{0}, y_{1}\right) \in\{0\} \times \mathcal{R}(k)$,

$$
\left\|y_{1}\right\|_{*}^{2} \leq C \int_{-\tilde{S}}^{\tilde{S}}\|B y(s)\|_{U}^{2} d s .
$$

Indeed, in that case, if $z_{0}=\sum_{j} a_{j} \Phi_{j} \in X$, using the transmutation technique of Theorem 2.1 with $S$ replaced by $\tilde{S}$, we obtain a solution $y$ of the wave 1.14 which initial data $y_{0}=0$ and

$\partial_{s} y(0)=\int_{0}^{\infty} \partial_{s} k(t, 0) z(t) d t=\frac{\tilde{S}}{4 \sqrt{\pi}} \sum a_{j} \Phi_{j} \int_{0}^{\infty} \frac{1}{t^{3 / 2}} \exp \left(-\frac{\tilde{S}^{2}}{4}-\mu_{j} t\right) d t$.

Applying (4.4), we immediately get, similarly as in Theorem 2.2 .

$$
\left\|\sum_{j} a_{j} \Phi_{j} \beta_{j}\right\|_{*}^{2} \leq C \int_{-\tilde{S}}^{\tilde{S}}\left\|\int_{0}^{\infty} k(t, s) B z(t) d t\right\|_{U}^{2} d s,
$$

where $z$ is the solution of 1.13 with initial data $z_{0}=\sum a_{j} \Phi_{j} \in X$.

Using estimate 2.19) and Lemma 2.3 with $T=1$, one can even get

$$
\left\|\sum_{j} a_{j} \Phi_{j} \beta_{j}\right\|_{*}^{2} \leq C \int_{0}^{\infty} \log ^{2}(t+2)\|B z(t)\|_{U}^{2} d t .
$$

However, getting rid of the logarithm in the right hand side of 4.6 requires more information on the norm $\|\cdot\|_{*}$. In particular, if the norm $\|\cdot\|_{*}$ is determined by a sequence $\omega_{j}$ by

$$
\left\|\sum_{j} a_{j} \Phi_{j} \beta_{j}\right\|_{*}^{2}=\sum_{j}\left|a_{j}\right|^{2} \beta_{j}^{2} \omega_{j}^{2}
$$

and $\beta_{j}^{2} \omega_{j}^{2} \geq \exp \left(-2 \mu_{j} T_{0}\right)$ for all $j \in \mathbb{N}$, for some $T_{0}$ large enough, then the logarithm in 4.6) can be removed easily. Actually, in this case, similarly as in Lemma 2.2, for some time $T_{1}$ one can prove that

$$
\left\|\sum_{j} a_{j} \Phi_{j} \beta_{j}\right\|_{*}^{2} \leq C \int_{0}^{T_{1}}\|B z(t)\|_{U}^{2} d t .
$$

An important remark is that, for 4.6 to be useful in practice, one should have a good understanding of the norm $\|\cdot\|_{*}$ on the spectral components, similarly as in (4.7) above. We will present such case below. But we should also emphasize that getting a norm $\|\cdot\|_{*}$ as in 4.7 for the observability property (4.4) of the waves would imply the spectral controllability of waves, a fact which is not known to hold in general, see Section 5 . 
Also note that if one wants to derive finite-time horizon estimates or estimates on the cost of controllability, one can also use the kernels $k_{T}$ solutions of (3.1) used in Section 3 and easily derive

$$
\left\|\sum_{j} a_{j} \Phi_{j} \beta_{j}\left(k_{T}\right)\right\|_{*}^{2} \leq C \int_{-\tilde{S}}^{\tilde{S}}\left\|\int_{0}^{T} k_{T}(t, s) B z(t) d t\right\|_{U}^{2} d s .
$$

Pointwise observation in dimension 1. Let $\Omega=(0,1), X=H^{-1}(\Omega), A=$ $-\Delta$ with domain $\mathcal{D}(A)=H_{0}^{1}(0,1)$.

Now, let $x_{0} \in(0,1)$ be such that $x_{0} \notin \mathbb{Q}$.

Define $B$ by $B z=z\left(x=x_{0}\right)$. As one can check, $B$ is continuous from $\mathcal{D}\left(A^{3 / 4+\varepsilon}\right)$ to $\mathbb{R}$ for any $\varepsilon>0$.

Solutions $y$ of the 1 -d wave equation with $y_{0}=0$ can be written as

$$
y(s, x)=\sum_{j=1}^{\infty} a_{j} \sin (j \pi x) \frac{1}{j \pi} \sin (j \pi s) .
$$

Hence, using Parseval's identity,

$$
\int_{-1}^{1}|B y(s)|^{2} d s=2 \sum_{j=1}^{\infty}\left|a_{j}\right|^{2} \frac{\sin ^{2}\left(j \pi x_{0}\right)}{j^{2} \pi^{2}} .
$$

Thus, since $x_{0} \notin \mathbb{Q}, 4.4$ holds with

$$
\left\|\sum_{j=1}^{\infty} a_{j} \sin (j \pi x)\right\|_{*}^{2}=\sum_{j=1}^{\infty}\left|a_{j}\right|^{2} \frac{\sin ^{2}\left(j \pi x_{0}\right)}{j^{2} \pi^{2}} .
$$

Therefore, one can conclude that, if $z$ solves the $1 \mathrm{~d}$ heat equation on $(0,1)$ with initial data $z_{0}(x)=\sum_{j=1}^{\infty} z_{j} \sin (j \pi x)$,

$$
\sum_{j=1}^{\infty}\left|z_{j}\right|^{2} \beta_{j}^{2} \frac{\sin ^{2}\left(j \pi x_{0}\right)}{j^{2} \pi^{2}} \leq C \int_{0}^{\infty}\left|z\left(t, x_{0}\right)\right|^{2} \log ^{2}(t+2) d t
$$

where the coefficients $\beta_{j}$ are given by 2.31 .

Using (2.32), this yields

$$
\sum_{j=1}^{\infty}\left|z_{j}\right|^{2} \exp (-2 \pi j) \frac{\sin ^{2}\left(j \pi x_{0}\right)}{j^{3} \pi^{3}} \leq C \int_{0}^{\infty}\left|z\left(t, x_{0}\right)\right|^{2} \log ^{2}(t+2) d t,
$$

Whether or not this result is sharp is an open problem. A priori, with no further assumption, whether or not the logarithmic dependence of time in the right hand-side is needed is not clear.

Note that, when $x_{0}$ is so that there exists $C>0$ such that for all $k \in \mathbb{N}$,

$$
\inf _{p \in \mathbb{Z}}\left\{\left|k x_{0}-p\right|\right\} \geq \frac{C}{k}
$$


(according to [19, p.124], the set of such $x_{0}$ is uncountable, thus non-empty) then

$$
\sin ^{2}\left(j \pi x_{0}\right) \geq \frac{C}{j^{2}}, \quad j \in \mathbb{N} \backslash\{0\} .
$$

One can then go further than (4.11), and prove, similarly as in Theorem 1.1 that for all $T>0$, there exists $C(T)$ such that

$$
\sum_{j=1}^{\infty}\left|z_{j}\right|^{2} \exp (-2 \pi j) \frac{\sin ^{2}\left(j \pi x_{0}\right)}{j^{3} \pi^{3}} \leq C \int_{0}^{T}\left|z\left(t, x_{0}\right)\right|^{2} d t .
$$

Using Remark 2.2 and especially equation 2.34), one can reformulate that estimate into the following form: for any $\gamma>1 / 2$, solutions of the 1 -d heat equation satisfy

$$
\int_{0}^{\infty} \int_{0}^{1} \exp \left(-\frac{\gamma}{t}\right)|z(t, x)|^{2} d t d x \leq C \int_{0}^{T}\left|z\left(t, x_{0}\right)\right|^{2} d t .
$$

Remark 4.1 Note that here the constant $\gamma$ does not seem to depend significantly on the position of $x_{0}$ on $(0,1)$. This is due to the fact that the technique used to prove (4.5) with the norm $\|\cdot\|_{*}$ in 4.9 is based on Fourier techniques. This is well-known, for instance, that direct applications of Ingham inequalities yield only observability in time 2 for the wave equation on $(0,1)$, even when observing from $(1 / 4,3 / 4)$, though such case is observable in any time larger than $1 / 2$.

The unit square observed from only one side. Here, we come back to the heat equation in the unit square $\Omega=(0,1)^{2}$, observed from one side of the boundary $\Gamma=\{0\} \times(0,1)$, see Figure 1 right.

In that case, the wave equation is not observable, since any vertical line corresponds to a trapped ray that does not meet the control region $\Gamma$. However, the solutions $y$ of the wave equation $(1.9)$ satisfy the following unique continuation property (Holmgren Uniqueness Theorem): For any $S>1$,

$$
\partial_{1} y\left(s, 0, x_{2}\right)=0, \quad\left(s, x_{2}\right) \in(-S, S) \times(-1,1) \quad \Rightarrow \quad y \equiv 0 .
$$

Here, and in what follows, we have denoted by $\partial_{1}$ the derivative with respect to $x_{1}$ to simplify the notations.

This unique continuation property indicates that the $L^{2}\left(-S, S ; L^{2}(0,1)\right)$ norm of $\partial_{1} y\left(s, 0, x_{2}\right)$ is a norm on the solutions $y$ of the waves for $S>1$. Of course, this does not provide any further information if we are not able to describe more precisely this norm, or any non-trivial weaker one $\|\cdot\|_{*}$ as in (4.4).

It turns out that in this geometric configuration $\Omega=(0,1)^{2}, \Gamma=\{0\} \times$ $(0,1)$, a norm $\|\cdot\|_{*}$ satisfying [4.4 can be derived explicitly (see [16]). For that to be done, write the solutions $y$ of $(1.9)$ as

$$
y\left(s, x_{1}, x_{2}\right)=\sqrt{2} \sum_{j=1}^{\infty} y_{j}\left(s, x_{1}\right) \sin \left(j \pi x_{2}\right) .
$$


This can be done of course since the functions $(x \mapsto \sin (j \pi x))_{j \in \mathbb{N}}$ form a basis of $L^{2}(0,1)$.

Then one immediately gets that

$$
\int_{-S}^{S} \int_{0}^{1}\left|\partial_{1} y\left(s, 0, x_{2}\right)\right|^{2} d x_{2} d s=\sum_{j=1}^{\infty} \int_{-S}^{S}\left|\partial_{1} y_{j}(s, 0)\right|^{2} d s
$$

and that

$$
\partial_{s s} y_{j}-\partial_{11} y_{j}+j^{2} \pi^{2} y_{j}=0, \quad s \in \mathbb{R}, j \in \mathbb{N}, \quad y_{j}(s, 0)=y_{j}(s, 1)=0 .
$$

In other words, using this decomposition, we decouple the contributions of each $y_{j}$.

Note that equation 4.18 is a simple $1 \mathrm{~d}$ wave equation with a potential. Hence, to prove its observability, we use the classical technique of lateral propagation of the energy (widely used in the context of $1 \mathrm{~d}$ semilinear wave equation, see e.g. [41). In our case, this reads as follows: For smooth solutions of 4.18, introduce the quantity

$$
F_{j}\left(x_{1}\right)=\int_{x_{1}-S}^{S-x_{1}}\left(\left|\partial_{s} y_{j}\left(s, x_{1}\right)\right|^{2}+\left|\partial_{1} y_{j}\left(s, x_{1}\right)\right|^{2}+j^{2} \pi^{2}\left|y_{j}\left(s, x_{1}\right)\right|^{2}\right) d s,
$$

and differentiate it. After straightforward computations, we get

$$
\begin{aligned}
\partial_{1} F_{j}\left(x_{1}\right) & \leq 2 \int_{x_{1}-S}^{S-x_{1}} \partial_{1} y_{j}\left(s, x_{1}\right)\left(-\partial_{s s} y_{j}\left(s, x_{1}\right)+\partial_{11} y_{j}\left(s, x_{1}\right)+j^{2} \pi^{2} y_{j}\left(s, x_{1}\right)\right) d s \\
& \leq 4 j^{2} \pi^{2} \int_{x_{1}-S}^{S-x_{1}} \partial_{1} y_{j}\left(s, x_{1}\right) y_{j}\left(s, x_{1}\right) d s \leq 2 j \pi F_{j}\left(x_{1}\right) .
\end{aligned}
$$

Hence we obtain, for any $S>1$, that $F_{j}\left(x_{1}\right) \leq \exp \left(2 j \pi x_{1}\right) F_{j}(0)$ for all $x_{1} \in(0,1)$. Integrating, we obtain

$$
\int_{0}^{1} F_{j}\left(x_{1}\right) d x_{1} \leq \frac{\exp (2 j \pi)}{2 j \pi} F_{j}(0)=\frac{\exp (2 j \pi)}{2 j \pi} \int_{-S}^{S}\left|\partial_{1} y_{j}(s, 0)\right|^{2} d s .
$$

But of course, the energy

$$
E_{j}(s)=\frac{1}{2} \int_{0}^{1}\left(\left|\partial_{s} y_{j}\left(s, x_{1}\right)\right|^{2}+\left|\partial_{1} y_{j}\left(s, x_{1}\right)\right|^{2}+j^{2} \pi^{2}\left|y_{j}\left(s, x_{1}\right)\right|^{2}\right) d x_{1}
$$

of $y_{j}$ solution of 4.18 is constant in time. Therefore, due to 4.19 , solutions $y_{j}$ of 4.18 satisfy, for any $S>1$,

$$
4 j \pi(S-1) E_{j}(0) \exp (-2 j \pi) \leq \int_{-S}^{S}\left|\partial_{1} y_{j}(s, 0)\right|^{2} d s .
$$

Using 4.16, 4.17, and estimates 4.20 for solutions of 4.18, we obtain (4.4) for any time $S>1$ with the norm $\|\cdot\|_{*}$ given by

$$
\left\|\sqrt{2} \sum_{j=1}^{\infty} y_{j}\left(x_{1}\right) \sin \left(j \pi x_{2}\right)\right\|_{*}^{2}=\sum_{j=1}^{\infty} j \exp (-2 \pi j) \int_{0}^{1}\left|y_{j}\left(x_{1}\right)\right|^{2} d x_{1} .
$$


Indeed, if $y$ is a solution of the wave (1.9) with initial data $y_{0}=0, y_{1}=$ $\sqrt{2} \sum_{j} y_{1, j}\left(x_{1}\right) \sin \left(j \pi x_{2}\right)$, the corresponding $y_{j}$ in 4.16 satisfy $y_{j}(s=0)=$ $0, \partial_{s} y_{j}(s=0)=y_{1, j}$, and the energy $E_{j}(0)$ respectively reduces to the $L^{2}(0,1)$-norm of $y_{1, j}$.

The norm $\|\cdot\|_{*}$ can then be easily written for functions expanded on the basis of the eigenfunctions of the Dirichlet Laplace operator:

$$
\left\|2 \sum_{j, \ell=1}^{\infty} a_{\ell, j} \sin \left(\ell \pi x_{1}\right) \sin \left(j \pi x_{2}\right)\right\|_{*}^{2}=\sum_{j=1}^{\infty} \sum_{\ell=1}^{\infty} j \exp (-2 \pi j)\left|a_{\ell, j}\right|^{2} .
$$

Due to the explicit form 4.22 of $\|\cdot\|_{*}$, we obtain that solutions $z$ of the heat equation with initial data

$$
z_{0}\left(x_{1}, x_{2}\right)=2 \sum_{\ell, j} a_{j, \ell} \sin \left(\ell \pi x_{1}\right) \sin \left(j \pi x_{2}\right)
$$

satisfy

$$
\begin{aligned}
\sum_{j=1}^{\infty} \sum_{\ell=1}^{\infty} j \exp (-2 \pi j) \mid & \left|a_{\ell, j}\right|^{2} \beta_{\ell, j}^{2} \\
& \leq C \int_{0}^{\infty} \int_{0}^{1} \log ^{2}(t+2)\left|\partial_{x} z\left(t, 0, x_{2}\right)\right|^{2} d x_{2} d s,
\end{aligned}
$$

where

$$
\beta_{\ell, j}=\int_{0}^{\infty} \frac{1}{t^{3 / 2}} \exp \left(-\frac{S^{2}}{4 t}-\pi^{2}\left(\ell^{2}+j^{2}\right) t\right) d t,
$$

which, according to (2.14, satisfy

$$
\beta_{\ell, j} \geq C \frac{\exp \left(-S \pi \sqrt{\ell^{2}+j^{2}}\right)}{\left(1+\pi \sqrt{\ell^{2}+j^{2}}\right)^{1 / 4}} .
$$

Using (4.23), we deduce

$$
\begin{aligned}
& \sum_{j=1}^{\infty} \sum_{\ell=1}^{\infty}\left|a_{\ell, j}\right|^{2} \frac{\exp \left(-2(S+1) \pi \sqrt{\ell^{2}+j^{2}}\right)}{(1+}\left.\pi \sqrt{\ell^{2}+j^{2}}\right)^{1 / 2} \\
& \leq C \int_{0}^{\infty} \int_{0}^{1} \log ^{2}(t+2)\left|\partial_{1} z\left(t, 0, x_{2}\right)\right|^{2} d x_{2} d t
\end{aligned}
$$

Of course, in (4.24), the norm in the left hand side is very similar to the one in the left hand-side of 2.10). Hence all the estimates done in Section 2.3 apply. In particular, for any $S>1$, we can get that, for any time $T>0$, there exists a constant $C(T)$ such that all the solutions $z$ of the heat equation (1.1) in the square $(0,1)^{2}$ satisfy:

$$
\int_{0}^{\infty} \exp \left(-\frac{(S+1)^{2}}{2 t}\right)\|z(t)\|_{L^{2}\left((0,1)^{2}\right.}^{2} d t \leq C \int_{0}^{T} \int_{0}^{1}\left|\partial_{x} z\left(t, 0, x_{2}\right)\right|^{2} d x_{2} d t .
$$


Note that estimate 4.25 yields an upper bound on $\gamma$ in 1.2 of the form $\gamma \leq(S+1)^{2} / 2=2^{+}$. This is still far away from the lower bound in (1.6)- 1.8 , which yield here $\tilde{\gamma} \geq 1 / 2$ and $\gamma \geq 1 / 8$.

Note that, using 29, Lemma 2.2] and 39, one can prove that inequality 1.3 holds in the square with $\tilde{\gamma}=3^{+}$. This, as we said, implies in particular that $\gamma \leq 3^{+}$in $(1.2)$ in this geometric configuration.

Though, our approach is developed directly on the weakly observable wave equation. To our knowledge, this is the first time that such case is addressed directly using a transmutation technique.

Note also that there should be some links with the estimates on the class of analyticity of functions that are controllable in time $T$ that have been derived in [3]. We also point out the recent work 24] which studies the observability problem in the square using biorthogonals.

Other examples. Let us also emphasize that our assumptions (4.4) are also satisfied in other non-trivial cases, and in particular on cylinders (2, 3]) and on networks of strings, for which under suitable assumptions (on the length of the strings and the pattern of the network) one can derive norms of the form 4.7 for which 4.4 holds, see 9].

\subsection{Arbitrary geometry}

Despite the previous examples, the arguments developed here do not seem to yield any explicit observability estimates for the heat equation out of the existing unique continuation results for the wave equation in general, except in some special geometries as the ones above, where a non-trivial norm $\|\cdot\|_{*}$ satisfying (4.4) can be made explicit.

In particular, situations in which the norm $\|\cdot\|_{*}$ has the form in (4.7) are particularly interesting. But this would imply (actually, these are equivalent) spectral controllability for the wave equation, a property which, to our knowledge, is not known so far in general geometries.

Also note that, when GCC fails, the existing unique continuation results on the wave equation refer to estimates in classes of analytic solutions (see [20]) or yield observability estimates depending on the frequency function of the solution (see [36,34]).

Let us also point out that, so far, there is no evidence of multi-dimensional situation in which the best observability constant $\gamma$ needs to be different from $\tilde{d}^{2} / 2$. The results of this paper show that in some cases, namely when $S=\tilde{d}=d$, this constant is sharp, but do not give any hint on those possible pathological situations.

\section{Further comments and open problems}

- The singular heat kernel. The equation 2.5 on $k$ is ill-posed. However, we have managed to find out an explicit solution. As mentioned in Section 3.1, in finite time horizons, such kernels $k_{T}$ solving (3.1) can be obtained using the classical construction by Tychonoff on non-standard heat 
kernels, see e.g. the textbook by F. John [17, and some other related ones as those in [18] and [13]. However, the estimates we have derived on the kernels functions $k_{T}$ solution of (3.1) do not seem to be optimal, thus explaining why our approach fails to provide sharp bounds on the finite time observability exponential cost.

- Lower bound for $\gamma$. In view of the results in [12, the lower bound (1.8) on $\gamma$ does not seem to be sharp. An interesting open question would be to improve this lower bound on $\gamma$. As we have seen, a reasonable conjecture would be $\gamma \geq \sup \left\{d\left(x_{0}, \omega\right)^{2} / 2, x_{0} \in \Omega\right\}$, but this is still an open problem so far.

Of course, that would in particular imply that the results given in Theorem 1.1 are sharp when $S=\tilde{d}$.

- Exponential observability and control cost for $T \sim 0$. The problem of characterizing the best constant $C(T)$ such that

$$
\int_{0}^{T} \exp \left(-\frac{S^{2}}{2 t}\right)\|z(t)\|_{X}^{2} d t \leq C(T) \int_{0}^{T}\|B z(t)\|_{U}^{2} d t
$$

holds is open. The constant $C(T)$ in $(5.1)$ is obviously bounded by the one obtained in (1.17), but the later is not explicit either when $T \sim 0$ since it has been deduced from a compactness argument. However, for times $T \geq T_{0}$ for $T_{0}$ large enough (see $(2.27)$ ), the constant $C(T)$ in $(1.17)$ and hence in (5.1) can be chosen to be $C\left(T_{0}\right)$, which can be made explicit, following the details of the proof of 2.28 .

One could expect the constant $C(T)$ in (5.1) not to blow up exponentially when $T$ goes to zero. If this were true, according to [31, we would obtain (1.3) with the optimal constant $\tilde{\gamma}=\left(S^{2} / 2\right)^{+}$but this is a widely open subject, as we have explained in Section 3.2. So far, the best constant in $(1.3)$ is $\tilde{\gamma}=\left(3 S^{2} / 2\right)^{+}$, as obtained in [39] (Note that [12] does not yield any estimate on $\tilde{\gamma}$, but only on $\gamma$ in 1.2 when considering the 1 -d heat equation observed from one boundary).

Observe also that, as pointed out in 43 and recently further developed in [27, these estimates and their optimality are intimately related with the spectral estimates by Lebeau and Robbiano [22] on the observability of packets of eigenfunctions.

- 1-d heat equations with rough coefficients. Our results apply for abstract heat and wave equations. Accordingly they can be used in the context of heat equations with variable coefficients as well. For instance, in one space dimension, as observed in [14, taking into account that sidewise energy estimates provide explicit observability constants for 1-d wave equations with $B V$ variable coefficients in an optimal characteristic time, one can obtain explicit observability estimates of the form 1.2 for the heat equation with $B V$ coefficients too. Note however that the 1-d heat equation is known to be observable for bounded measurable coefficients (1]). But that case cannot be treated by transmutation since the optimal assumption for the wave equation being observable is the $B V$-regularity of the coefficients ( 6 ). Therefore it needs to be treated directly. As far as we know, the obtention of sharp estimates on the observability constant is widely open in that case.

- On the GCC condition. 
1. Our result applies and yields a sharp observability constant under the GCC and when $S$ can be taken to be arbitrarily close to $d$. However, there are cases in which GCC holds but in a time $S$ much larger than $d$ and others in which GCC simply does not hold. Whether the lower bound $\sqrt{1.8}$ is sharp in those cases is an interesting open problem.

2. In the absence of GCC the wave equation satisfies a unique continuation property (Holmgren's uniqueness theorem) stating that the observation measures some very weak norm on the data. For instance, observability may hold within the class of solutions generated by a finite number of eigenfunctions but with an observability constant that depends exponentially on the frequency function (see $[21,36,35,34$ ).

In that case, using the recent results in [34], our transmutation technique and the iteration argument developed in 27] (see also [22] for the origin of this idea), one can derive observability estimates for the heat equation. This will be published in [11.

However, the weak observability estimates developed in 34 are given with constants that are not given explicitly in term of the geometry, thus yielding another question: Can we estimate precisely the constants coming into play within the quantification of the unique continuation property for the wave equation without GCC?

Another important question in that context is to understand whether or not spectral controllability for the wave equation holds for the wave equation in general geometry. To our knowledge, this is still an open problem, see [36, 34 for some partial results in that direction.

- Higher order parabolic equations. Our results also apply to the case of higher order parabolic equations. For instance, the plate equation, given by

$$
\begin{cases}\partial_{s s} y+\Delta^{2} y=0, & (s, x) \in \mathbb{R} \times \Omega, \\ y=\Delta y=0, & (s, x) \in \mathbb{R} \times \partial \Omega,\end{cases}
$$

is known to be observable through $\omega$ in any time $2 S>0$ when GCC holds in some time $\mathcal{S}_{0}>0$ (see, e.g., [23]).

Thus, in view of the results of the present paper, assuming that $\left(\omega, \Omega, \mathcal{S}_{0}\right)$ satisfies the GCC for some finite $\mathcal{S}_{0}$, for any $\gamma>0$, there exists a constant $C_{\gamma}$ such that solutions $z$ of

$$
\begin{cases}\partial_{t} z+\Delta^{2} z=0, & (t, x) \in \mathbb{R}_{+} \times \Omega \\ z=\Delta z=0, & (t, x) \in \mathbb{R} \times \partial \Omega\end{cases}
$$

satisfy

$$
\int_{0}^{\infty} \exp \left(-\frac{\gamma}{t}\right)\|z(t)\|_{X}^{2} d t \leq C_{\gamma} \int_{0}^{\infty}\|B z(t)\|_{U}^{2} d t .
$$

The fact that $\gamma$ can be chosen arbitrarily small reflects that, very likely, in this case, the observability inequality can be obtained with a less degenerate weight function. Note that in the case of the heat equation the weights of the form $\exp (-\gamma / t)$ are optimal because of the scaling of the heat kernel.

This also shows the impossibility of getting observability properties for the heat equation out of those on the Schrödinger equation by the methods in this paper. Indeed, the observability of the Schrödinger equation is equivalent 
to that of the plate equation (see 21]) and the later, as mentioned above, leads to the observability of the fourth order parabolic equation but not to the heat one.

- Fractional order parabolic equations. One could try to apply the same method to fractional order parabolic equations of the form:

$$
\begin{cases}\partial_{t} z+(-\Delta)^{\alpha} z=0, & (t, x) \in \mathbb{R} \times \Omega, \\ z=0, & (t, x) \in \mathbb{R} \times \partial \Omega,\end{cases}
$$

with $0<\alpha<1$. This equation is well known to be null controllable for $\alpha>1 / 2$ and the control property to fail for $\alpha \leq 1 / 2$ ([25, 32]).

But our transmutation method does not apply in this case in the sense that, even if one can write the solutions of this system in terms of those of the corresponding wave-like equation

$$
\begin{cases}\partial_{s s} y+(-\Delta)^{\alpha} y=0, & (s, x) \in \mathbb{R} \times \Omega, \\ y=0, & (s, x) \in \mathbb{R} \times \partial \Omega,\end{cases}
$$

the later fails to be controllable for $\alpha<1$. This can be easily seen in one space dimension by analyzing the spectrum of the fractional power of the Laplacian that shows a growth of order $\lambda(n) \sim C n^{2 \alpha}$ as $n \rightarrow \infty$ so that the classical uniform gap condition for $\sqrt{\lambda(n)} \sim C^{1 / 2} n^{\alpha}$, which is sharp for the control of 1-d wave models of this form by means of Ingham type inequalities, fails.

This is a further example of the fact that there are control results in the parabolic context that cannot be obtained from the hyperbolic one by means of transmutation.

Acknowledgements The authors thank Sorin Micu, Luc Miller, Luc Robbiano and Kim Dang Phung for interesting discussions and comments related to this work.

\section{References}

1. G. Alessandrini and L. Escauriaza. Null-controllability of one-dimensional parabolic equations. ESAIM Control Optim. Calc. Var., 14(2):284-293, 2008.

2. B. Allibert. Contrôle analytique de l'équation des ondes sur des surfaces de révolution. C. R. Acad. Sci. Paris Sér. I Math., 322(9):835-838, 1996.

3. B. Allibert. Analytic controllability of the wave equation over a cylinder. ESAIM Control Optim. Calc. Var., 4:177-207 (electronic), 1999.

4. C. Bardos, G. Lebeau, and J. Rauch. Sharp sufficient conditions for the observation, control and stabilization of waves from the boundary. SIAM J. Control and Optim., 30(5):1024-1065, 1992.

5. N. Burq and P. Gérard. Condition nécessaire et suffisante pour la contrôlabilité exacte des ondes. C. R. Acad. Sci. Paris Sér. I Math., 325(7):749-752, 1997.

6. C. Castro and E. Zuazua. Concentration and lack of observability of waves in highly heterogeneous media. Arch. Ration. Mech. Anal., 164(1):39-72, 2002.

7. X.-Y.Chen. A strong unique continuation theorem for parabolic equations. Math. Ann., 311(4):603-630, 1998.

8. J.-M. Coron and S. Guerrero. Local null controllability of the two-dimensional Navier-Stokes system in the torus with a control force having a vanishing component. J. Math. Pures Appl. (9), 92(5):528-545, 2009. 
9. R. Dáger and E. Zuazua. Wave propagation, observation and control in 1-d flexible multi-structures, volume 50 of Mathématiques 83 Applications (Berlin). Springer-Verlag, Berlin, 2006.

10. S. Ervedoza and E. Zuazua. A systematic method for building smooth controls for smooth data. Discrete Contin. Dyn. Syst. Ser. B, 14(4):1375-1401, 2010.

11. S. Ervedoza and E. Zuazua. Observability of heat processes by transmutation without geometric restrictions. Mathematical Control and Related Fields, to appear.

12. H. O. Fattorini and D. L. Russell. Exact controllability theorems for linear parabolic equations in one space dimension. Arch. Rational Mech. Anal., 43:272-292, 1971.

13. E. Fernández-Cara and E. Zuazua. The cost of approximate controllability for heat equations: the linear case. Adv. Differential Equations, 5(4-6):465-514, 2000.

14. E. Fernández-Cara and E. Zuazua. On the null controllability of the onedimensional heat equation with BV coefficients. Comput. Appl. Math., 21(1):167-190, 2002. Special issue in memory of Jacques-Louis Lions.

15. A. V. Fursikov and O. Y. Imanuvilov. Controllability of evolution equations, volume 34 of Lecture Notes Series. Seoul National University Research Institute of Mathematics Global Analysis Research Center, Seoul, 1996.

16. A. Haraux. A generalized internal control for the wave equation in a rectangle. J. Math. Anal. Appl., 153(1):190-216, 1990.

17. F. John. Partial differential equations, volume 1 of Applied Mathematical Sciences. Springer-Verlag, New York, fourth edition, 1982.

18. B. Frank Jones, Jr. A fundamental solution for the heat equation which is supported in a strip. J. Math. Anal. Appl., 60(2):314-324, 1977.

19. S. Lang. Introduction to diophantine approximations. Addison-Wesley Publishing Co., Reading, Mass.-London-Don Mills, Ont., 1966.

20. G. Lebeau. Contrôle analytique. I. Estimations a priori. Duke Math. J., 68(1):1-30, 1992.

21. G. Lebeau. Contrôle de l'équation de Schrödinger. J. Math. Pures Appl. (9), 71(3):267-291, 1992.

22. G. Lebeau and L. Robbiano. Contrôle exact de l'équation de la chaleur. Comm. Partial Differential Equations, 20(1-2):335-356, 1995.

23. J.-L. Lions. Contrôlabilité exacte, Stabilisation et Perturbations de Systèmes Distribués. Tome 1. Contrôlabilité exacte, volume RMA 8. Masson, 1988.

24. S. Micu and L. de Teresa. A spectral study of the boundary controllability of the linear 2-D wave equation in a rectangle. Asymptot. Anal., 66(3-4):139-160, 2010.

25. S. Micu and E. Zuazua. On the controllability of a fractional order parabolic equation. SIAM J. Control Optim., 44(6):1950-1972 (electronic), 2006.

26. S. Micu and E. Zuazua. Regularity issues for the null-controllability of the linear 1-d heat equation. Preprint, 2010.

27. L. Miller. A direct Lebeau-Robbiano strategy for the observability of heat-like semigroups. 21 pages. MSC: 93B07; 35B37.

28. L. Miller. Geometric bounds on the growth rate of null-controllability cost for the heat equation in small time. J. Differential Equations, 204(1):202-226, 2004.

29. L. Miller. On the null-controllability of the heat equation in unbounded domains. Bull. Sci. Math., 129(2):175-185, 2005.

30. L. Miller. The control transmutation method and the cost of fast controls. SIAM J. Control Optim., 45(2):762-772 (electronic), 2006.

31. L. Miller. On exponential observability estimates for the heat semigroup with explicit rates. Atti Accad. Naz. Lincei Cl. Sci. Fis. Mat. Natur. Rend. Lincei (9) Mat. Appl., 17(4):351-366, 2006.

32. L. Miller. On the controllability of anomalous diffusions generated by the fractional Laplacian. Math. Control Signals Systems, 18(3):260-271, 2006.

33. A. Münch and E. Zuazua. Numerical approximation of null controls for the heat equation through transmutation. to appear in Inverse Problems, 2010. 
34. K. D. Phung. Waves, damped wave and observation. In Ta-Tsien Li, YueJun Peng, and Bo-Peng Rao, editors, Some Problems on Nonlinear Hyperbolic Equations and Applications, Series in Contemporary Applied Mathematics CAM 15, 2010.

35. J. Rauch, X. Zhang, and E. Zuazua. Polynomial decay for a hyperbolicparabolic coupled system. J. Math. Pures Appl. (9), 84(4):407-470, 2005.

36. L. Robbiano. Fonction de coût et contrôle des solutions des équations hyperboliques. Asymptotic Anal., 10(2):95-115, 1995.

37. D. L. Russell. A unified boundary controllability theory for hyperbolic and parabolic partial differential equations. Studies in Appl. Math., 52:189-211, 1973.

38. T. I. Seidman. Time-invariance of the reachable set for linear control problems. J. Math. Anal. Appl., 72(1):17-20, 1979.

39. G. Tenenbaum and M. Tucsnak. New blow-up rates for fast controls of Schrödinger and heat equations. J. Differential Equations, 243(1):70-100, 2007.

40. D. V. Widder. The role of the Appell transformation in the theory of heat conduction. Trans. Amer. Math. Soc., 109:121-134, 1963.

41. E. Zuazua. Exact controllability for semilinear wave equations in one space dimension. Ann. Inst. H. Poincaré Anal. Non Linéaire, 10(1):109-129, 1993.

42. E. Zuazua. Some results and open problems on the controllability of linear and semilinear heat equations. In Carleman estimates and applications to uniqueness and control theory (Cortona, 1999), volume 46 of Progr. Nonlinear Differential Equations Appl., pages 191-211. Birkhäuser Boston, Boston, MA, 2001.

43. E. Zuazua. Controllability and observability of partial differential equations: Some results and open problems. In C.M. Dafermos and Elsevier Science E. Feireisl eds, editors, Handbook of Differential equations, volume 3, pages $527-621,2006$ 